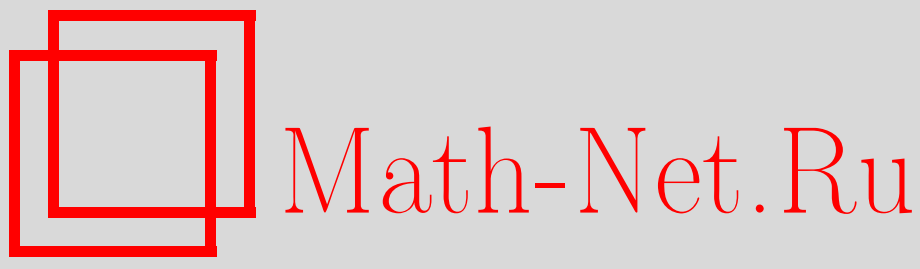

В. М. Круглов, Асимптотическое поведение статистики Пирсона, Теория вероятн. и ее примен., 2000, том 45, выпуск $1,73-102$

DOI: https://doi.org/10.4213/tvp325

Использование Общероссийского математического портала Math-Net.Ru подразумевает, что вы прочитали и согласны с пользовательским соглашением http://www . mathnet.ru/rus/agreement

Параметры загрузки:

IP: 3.82 .47 .9

26 апреля 2023 г., 02:58:05

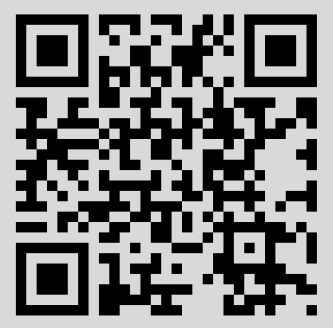




\title{
АСИМПТОТИЧЕСКОЕ ПОВЕДЕНИЕ СТАТИСТИКИ ПИРСОНА ${ }^{1)}$
}

\begin{abstract}
Доказан ряд предельных теорем для некоторых функционалов от статистики Пирсона, построенной по полиномиальному распределению с параметрами $n$ и $p_{k}, k=1,2, \ldots, s=s(n)$, в предположении, что $\inf _{n}\left\{n \min _{1 \leqslant k \leqslant s} p_{k}\right\}>0, s \rightarrow \infty, n \min \left\{p_{k}: k \in W_{n}\right\} \rightarrow \infty$, $N_{n} / s \rightarrow 1$ при $n \rightarrow \infty$, где $N_{n}$ - число элементов в множестве $W_{n} \subset\{1,2, \ldots, s\}$. В частности, доказаны многомерная и функциональная предельные теоремы для этой статистики. В целом доказанные в статье утверждения показывают, что статистика Пирсона ведет себя во многих отношениях как асимптотически нормальная сумма независимых случайных величин.
\end{abstract}

Ключевые слова и фразы: статистика Пирсона, статистика хи-квадрат, случайные ломаные, полиномиальное распределение.

1. Введение. Статистика Пирсона, ее также называют статистикой хи-квадрат, была введена в употребление $\mathrm{K}$. Пирсоном в связи $\mathrm{c}$ решением ряда задач математической статистики. С многочисленными применениями этой статистики можно познакомиться, например, по учебнику [1]. Нас интересуют асимптотические свойства статистики Пирсона. Имеется большое число публикаций, посвященных исследованию асимптотических свойств самой статистики Пирсона и многочисленных ее модификаций (см. [1]-[6], [14]-[17] и литературу, указанную там). Такие статистики, как правило, представляют собой суммы зависимых случайных величин. Для доказательства предельных теорем для таких сумм был выработан специальный аппарат. Исключением является статья С. X. Туманяна [2], где осуществлен прямой анализ статистики Пирсона. В предлагаемой статье показано, что метод, использованный Туманяном, позволяет доказать ряд интересных обобщений его теоремы. Из шести теорем, доказанных ниже, первые три теоремы представляют собой своего рода обобщения теоремы Туманяна. Остальные три теоремы доказаны с привлечением некоторых соображений из статьи автора [7]. Наша теорема 3 напоминает теорему из статьи [17], где

${ }^{*}$ МГУ, Факультет вычислительной математики и кибернетики, Воробьевы горы, 119899 Москва, Россия.

1) Работа выполнена при поддержке Российского фонда фундаментальных исследований, гранты 99-01-00846, 99-01-00847. 
получено предельное совместное распределение конечного числа статистик $\chi^{2}$, вычисленных по нарастающим объемам выборок (при фиксированном числе исходов в соответствующих полиномиальных схемах).

Пусть на вероятностном пространстве $(\Omega, \mathscr{F}, \mathbf{P})$ определен случайный вектор $\bar{\nu}=\left(\nu_{1}, \nu_{2}, \ldots, \nu_{s}\right)$ с полиномиальным распределением. В соответствии с определением полиномиального распределения мы имеем

$$
\mathbf{P}\left\{\nu_{1}=m_{1}, \nu_{2}=m_{2}, \ldots, \nu_{s}=m_{s}\right\}=\frac{n !}{m_{1} ! m_{2} ! \cdots m_{s} !} p_{1}^{m_{1}} p_{2}^{m_{2}} \cdots p_{s}^{m_{s}}
$$

для любых неотрицательных целых чисел $m_{1}, m_{2}, \ldots, m_{s}$, сумма которых равна $n$, где $p_{k}>0, k=1,2, \ldots, s$, и сумма этих чисел равна единице.

Статистикой Пирсона называется случайная величина

$$
\chi_{n}^{2}=\sum_{k=1}^{s} \frac{n}{p_{k}}\left(\frac{\nu_{k}}{n}-p_{k}\right)^{2}
$$

Легко видеть, что случайная величина $\nu_{k}$ имеет биномиальное распределение с параметрами $n$ и $p_{k}$. Обозначим $q_{k}=1-p_{k}$. Стандартные вычисления приводят к следующим выражениям для математических ожиданий, дисперсий и ковариаций слагаемых суммы (1):

$$
\begin{aligned}
& \mathbf{E}\left(\frac{n}{p_{k}}\left(\frac{\nu_{k}}{n}-p_{k}\right)^{2}\right)=q_{k} \\
& \mathbf{D}\left(\frac{n}{p_{k}}\left(\frac{\nu_{k}}{n}-p_{k}\right)^{2}\right)=2 q_{k}^{2}+\frac{q_{k}}{n p_{k}}\left(1-6 p_{k} q_{k}\right) \\
& \operatorname{Cov}\left(\frac{n}{p_{k}}\left(\frac{\nu_{k}}{n}-p_{k}\right)^{2}, \frac{n}{p_{j}}\left(\frac{\nu_{j}}{n}-p_{j}\right)^{2}\right) \\
& \quad=2 p_{k} p_{j}+\frac{1}{n}\left(2 p_{k}+2 p_{j}-6 p_{k} p_{j}\right)-\frac{1}{n}, \quad k \neq j
\end{aligned}
$$

С помощью этих формул нетрудно найти известные выражения для математического ожидания и дисперсии статистики Пирсона

$$
\mathbf{E} \chi_{n}^{2}=s-1, \quad \mathbf{D} \chi_{n}^{2}=2(s-1)+\frac{1}{n}\left(\sum_{k=1}^{s} \frac{1}{p_{k}}-s^{2}-2 s+2\right)
$$

Теорема Туманяна. Если величины $s$ u $p_{k}, k=1,2, \ldots, s$, зав исят от $n$ таким образом, что $s \rightarrow \infty n$ ри $n \rightarrow \infty u \lim _{n \rightarrow \infty} n \min _{1 \leqslant k \leqslant s} p_{k}=$ $\infty$, mo

$$
\lim _{n \rightarrow \infty} \mathbf{P}\left\{\frac{\chi_{n}^{2}-s}{\sqrt{2 s}}<x\right\}=\Phi(x)=\frac{1}{\sqrt{2 \pi}} \int_{-\infty}^{x} e^{-u^{2} / 2} d u, \quad x \in \mathbf{R}^{1}
$$


Д о к а з т е л ь с в о см. в [2]. Если цитировать теорему Туманяна буквально, то вместо $s$ в (4) следовало бы писать $s-1$. Ясно, что обе записи эквивалентны.

Свойства полиномиального распределения позволяют совсем просто усилить теорему Туманяна. Пусть дано подмножество $W$ множества $\{1,2, \ldots, s\}$. Мы предполагаем, что входяпие в $W$ иисла записаны в виде конечной последовательности в возрастающем порядке. Обозначим $\bar{W}=$ $\{1,2, \ldots, s\} \backslash W, N(W)$ - число элементов в $W, p_{0}(W)=\min \left\{p_{k}: k \in W\right\}$,

$$
\chi_{n}^{2}(W)=\sum_{k \in W} \frac{n}{p_{k}}\left(\frac{\nu_{k}}{n}-p_{k}\right)^{2}
$$

Теорема 1. Iредположим, ито величины $s$ u $p_{k}, k=1,2, \ldots, s, u$ множество $W$ завислт от $n$ тахим образом, ито $s \rightarrow \infty$ nрu $n \rightarrow \infty u$

$$
\begin{gathered}
\inf _{n}\left\{n \min _{1 \leqslant k \leqslant s} p_{k}\right\}=\alpha>0, \\
\lim _{n \rightarrow \infty} n p_{0}(W)=\infty \\
\lim _{n \rightarrow \infty} \frac{1}{s} N(W)=1
\end{gathered}
$$

Тогда выполняется (4), что также можно записать в эквивалентной форме

$$
\lim _{n \rightarrow \infty} \mathbf{P}\left\{\frac{\chi_{n}^{2}-\mathbf{E} \chi_{n}^{2}}{\sqrt{\mathbf{D} \chi_{n}^{2}}}<x\right\}=\Phi(x) .
$$

Д ок а з а т ль с т о. Без ограничения общности рассуждений можно считать, что $W=\{r, r+1, \ldots, l+1\}$ для некоторых $r$ и $l, 1 \leqslant r<$ $l<s$. Положим

$$
\begin{aligned}
p_{r, k} & =p_{k}, \quad \nu_{r, k}=\nu_{k}, \quad k=r, r+1, \ldots, l \\
p_{r, l+1} & =1-p_{r}-\cdots-p_{l}, \quad \nu_{r, l+1}=n-\nu_{r}-\nu_{r+1}-\cdots-\nu_{r}
\end{aligned}
$$

Случайный вектор $\left(\nu_{r, r}, \ldots, \nu_{r, l+1}\right)$ имеет полиномиальное распределение, так как для любых неотрицательных целых чисел $m_{r}, \ldots, m_{l+1}$, $m_{r}+m_{r+1}+\cdots+m_{l+1}=n$,

$$
\mathbf{P}\left\{\nu_{r, r}=m_{r}, \ldots, \nu_{r, l+1}=m_{l+1}\right\}=\frac{n !}{m_{r} ! \cdots m_{l+1} !} p_{r, r}^{m_{r}} \cdots p_{r, l+1}^{m_{l+1}}
$$

Запишем соответствуюшую статистику Пирсона в виде суммы двух слаraembx

$$
\sum_{k=r}^{l+1} \frac{n}{p_{r, k}}\left(\frac{\nu_{r, k}}{n}-p_{r, k}\right)^{2}=\chi_{n}^{2}(r, l)+\frac{n}{p_{r, l+1}}\left(\frac{\nu_{r, l+1}}{n}-p_{r, l+1}\right)^{2},
$$


где

$$
\chi_{n}^{2}(r, l)=\chi_{n}^{2}\left(W^{\prime}\right), \quad W^{\prime}=\{r, r+1, \ldots, l\} .
$$

В силу (8) $N\left(W^{\prime}\right)=(l+1-r) \rightarrow \infty$. Условие (7) позволяет применить к этой статистике теорему Туманяна, согласно которой

$$
\lim _{n \rightarrow \infty} \mathbf{P}\left\{\sum_{k=r}^{l+1} \frac{n}{p_{r, k}}\left(\frac{\nu_{r, k}}{n}-p_{r, k}\right)^{2}-(l+2-r)<x \sqrt{2(l+2-r)}\right\}=\Phi(x),
$$

и, следовательно,

$$
\begin{gathered}
\lim _{n \rightarrow \infty} \mathbf{P}\left\{\sum_{k=r}^{l} \frac{n}{p_{r, k}}\left(\frac{\nu_{r, k}}{n}-p_{r, k}\right)^{2}-(l+2-r)\right. \\
<x \sqrt{2(l+2-r)}\}=\Phi(x),
\end{gathered}
$$

так как $\lim _{n \rightarrow \infty} \mathbf{P}\left\{n / p_{r, l+1}\left(\nu_{r, l+1} / n-p_{r, l+1}\right)^{2}>\varepsilon \sqrt{2(l+2-r)}\right\}=0, \varepsilon>0$. Действительно, по неравенству Маркова вероятность, стоящая под знаком предела, не превосходит величины

$$
\mathbf{E}\left(\frac{n}{p_{r, l+1}}\left(\frac{\nu_{r, l+1}}{n}-p_{r, l+1}\right)^{2}\right) \frac{1}{\varepsilon \sqrt{2(l+2-r)}}=\frac{1-p_{r, l+1}}{\varepsilon \sqrt{2(l+2-r)}},
$$

стремящейся к нулю при $l+2-r \rightarrow \infty$. Точно так же докажем, что

$$
\lim _{n \rightarrow \infty} \mathbf{P}\left\{\frac{n}{p_{l+1}}\left(\frac{\nu_{l+1}}{n}-p_{l+1}\right)^{2}>\varepsilon \sqrt{2(l+2-r)}\right\}=0, \quad \varepsilon>0 .
$$

Отсюда и из (12) следует

$$
\lim _{n \rightarrow \infty} \mathbf{P}\left\{\frac{\chi_{n}^{2}(W)-N(W)}{\sqrt{2 N(W)}}<x\right\}=\Phi(x), \quad x \in \mathbf{R}^{1},
$$

так как $N(W)=l+2-r$.

Докажем, что

$$
\lim _{n \rightarrow \infty} \mathbf{P}\left\{\left|\frac{\chi_{n}^{2}(\bar{W})-N(\bar{W})}{\sqrt{2 s}}\right|>\varepsilon\right\}=0, \quad \varepsilon>0 .
$$

Можно считать, что $s>\frac{1}{2} \varepsilon^{-2}$. Вероятность события в (14) не превосходит величины

$$
\mathbf{P}\left\{\left|\frac{\chi_{n}^{2}(\bar{W})-\mathbf{E} \chi_{n}^{2}(\bar{W})}{\sqrt{2 s}}\right|>\varepsilon-\frac{1}{\sqrt{2 s}}\right\}=0
$$


Tak Kak

$$
N(\bar{W})=\mathbf{E} \chi_{n}^{2}(\bar{W})+\sum_{k \in \bar{W}} p_{k}
$$

Последняя вероятность по неравенству Чебышева не превосходит $\mathrm{D} \chi_{n}^{2}(\bar{W}) / 2 s(\varepsilon-1 / \sqrt{2 s})$. По приведенным выше формулам для вычисления дисперсий и ковариаций слагаемых суммы (5) мы получим

$$
\begin{aligned}
\mathbf{D} \chi_{n}^{2}(\bar{W})= & \sum_{k \in \bar{W}}\left(2 q_{k}^{2}+\frac{q_{k}}{n p_{k}}\left(1-6 p_{k} q_{k}\right)\right) \\
& +\sum_{k, j \in \bar{W}, k \neq j}\left(2 p_{k} p_{j}+\frac{1}{n}\left(2 p_{k}+2 p_{j}-6 p_{k} p_{j}\right)-\frac{1}{n}\right) \\
\leqslant & \left(6+\frac{7}{\alpha}+\frac{8}{n}\right) N(\bar{W}),
\end{aligned}
$$

и, следовательно, в силу (8), $\mathbf{D} \chi_{n}^{2}(\bar{W}) / 2 s \longrightarrow 0$ при $n \rightarrow \infty$. Соотношение (14) доказано. Утверждение (4) следует из (13), (14) и условия (8), если учесть равенство $N(W)+N(\bar{W})=s$. Чтобы завершить доказательство, нам осталось убедиться, что (4) и (9) эквивалентны. В виду равенства $\mathbf{E} \chi_{n}^{2}=s-1$, для этого достаточно доказать, что

$$
\lim _{n \rightarrow \infty} \frac{1}{2 s} \mathbf{D} \chi_{n}^{2}=1
$$

Из очевидных неравенств

$$
N(W) p_{0}(W) \leqslant \sum_{k=1}^{s} p_{k}=1, \quad n p_{0}(W) \leqslant \frac{n}{N(W)}
$$

и условий (7) и (8) следует, что $s / n \rightarrow 0$ при $n \rightarrow \infty$. Запишем формулу (3) для дисперсии $\chi_{n}^{2}$ в следующем виде: $\mathrm{D} \chi_{n}^{2}=2 s((s-1) / s+R)$, где

$$
\begin{aligned}
R & =\frac{1}{2 s n}\left(\sum_{k \in W} \frac{1}{p_{k}}+\sum_{k \in \bar{W}} \frac{1}{p_{k}}\right)-\frac{s}{2 n}+\frac{2-2 s}{2 s n} \\
& \leqslant \frac{N(W)}{2 s n p_{0}(W)}+\frac{N(\bar{W})}{2 s \alpha}-\frac{s}{2 n}+\frac{1-s}{s n}
\end{aligned}
$$

На основании (7) и (8) мы заключаем, что $R \rightarrow 0$ при $n \rightarrow \infty$. Теорема доказана.

При доказательстве теоремы 1 мы попутно доказали еще одно усиление теоремы Туманяна. 
Теорема 2. Предположим, что множество $W$ и величины $p_{k}$, $k \in W$, зависят от $n$ таким образом, ито $N(W) \rightarrow \infty$ nри $n \rightarrow \infty$ и выполнено условие (7). Тогда выполняется (13), ито тажже можно переписать в следующей эквивалентной форме:

$$
\lim _{n \rightarrow \infty} \mathbf{P}\left\{\frac{\chi_{n}^{2}(W)-\mathbf{E} \chi_{n}^{2}(W)}{\sqrt{\mathbf{D} \chi_{n}^{2}(W)}}<x\right\}=\Phi(x), \quad x \in \mathbf{R}^{1} .
$$

Д ок а з а т е л с т в о. Утверждение (13) было доказано при доказательстве предыдущей теоремы. В виду равенства $N(W)=$ $\mathbf{E} \chi_{n}^{2}(W)+\sum_{k \in W} p_{k}$ эквивалентность (13) и (17) следует из соотношения

$$
\lim _{n \rightarrow \infty} \frac{1}{2 N(W)} \mathbf{D} \chi_{n}^{2}(W)=1 .
$$

Докажем (18). По формулам для вычисления дисперсий и ковариаций слагаемых суммы (5) мы получим

$$
\begin{aligned}
\mathbf{D} \chi_{n}^{2}(W)= & \sum_{k \in W}\left(2 q_{k}^{2}+\frac{q_{k}}{n p_{k}}\left(1-6 p_{k} q_{k}\right)\right)+ \\
& +\sum_{k, j \in W, k \neq j}\left(2 p_{k} p_{j}+\frac{1}{n}\left(2 p_{k}+2 p_{j}-6 p_{k} p_{j}\right)-\frac{1}{n}\right) .
\end{aligned}
$$

Из (7) и (16) следует, что $N(W) / n \rightarrow 0$ при $n \rightarrow \infty$. Отсюда и из соотношений

$$
\begin{gathered}
\sum_{k \in W} 2 q_{k}^{2}=2 N(W)-4 \sum_{k \in W} p_{k}+2 \sum_{k \in W} p_{k}^{2}, \\
\left|\sum_{k \in W} \frac{q_{k}}{n p_{k}}\left(1-6 p_{k} q_{k}\right)\right| \leqslant \frac{7 N(W)}{n p_{0}(W)} \\
\left|\sum_{k, j \in W, k \neq j}\left(2 p_{k} p_{j}+\frac{1}{n}\left(2 p_{k}+2 p_{j}-6 p_{k} p_{j}\right)-\frac{1}{n}\right)\right| \\
\leqslant 2+\frac{4}{n} N(W)+\frac{6}{n}+\frac{(N(W))^{2}}{n}
\end{gathered}
$$

следует (18), если принять во внимание условие (7). Теорема доказана.

Теперь мы проведем некоторые преобразования над случайными величинами, что облегчит нам доказательство основного результата следующего пункта. Преобразуем сумму, стоящую в левой части (11). Положим

$\rho_{r, k}=\frac{\nu_{r, k}}{n}-p_{r, k}, \quad \pi_{r, k}=p_{r, r}+p_{r, r+1}+\cdots+p_{r, k}, \quad k=r, r+1, \ldots, l+1$.

Выразим случайные величины $\rho_{r, k}, k=r, r+1, \ldots, l+1$, через новые случайные величины $y_{r, 0}=0, y_{r, k}, k=r, r+1, \ldots, l$, посредством линейного 
преобразования

$\rho_{r, k}=-\pi_{r, k-1} y_{r, k-1}+p_{r, k} \sum_{i=k}^{l} y_{r, i}, \quad k=r, r+1, \ldots, l, \quad \rho_{r, l+1}=-\pi_{r, l} y_{r, l}$.

Легко проверить, что

$$
\sum_{k=r}^{l+1} \frac{n}{p_{r, k}}\left(\frac{\nu_{r, k}}{n}-p_{r, k}\right)^{2}=\sum_{k=r}^{l+1} \frac{n}{p_{r, k}} \rho_{r, k}^{2}=\sum_{k=r}^{l} \frac{n \pi_{r, k} \pi_{r, k+1}}{p_{r, k+1}} y_{r, k}^{2}
$$

Далее нам понадобятся выражения случайных величин $y_{r, k}$ через случайные величины $\rho_{r, k}$. С этой целью заметим, что уравнения, связываюшие их, линейно зависимы, так как суммирование уравнений системы приводит к равенству $0=0$. Напротив, частичная система уравнений

$$
\begin{aligned}
\rho_{r, k} & =-\pi_{r, k-1} y_{r, k-1}+p_{r, k} \sum_{i=k}^{l} y_{r, i}, \quad k=r+1, r+2, \ldots, l \\
\rho_{r, l+1} & =-\pi_{r, l} y_{r, l},
\end{aligned}
$$

является определенной. Ее матрица имеет треугольный вид. Определитель матрицы равен произведению $(-1)^{l} \pi_{r, r} \pi_{r, r+1} \cdots \pi_{r, l}$ и, следовательно, отличен от нуля. Поэтому для вычисления $y_{r, k}$ можно применить известное правило Крамера $[8$, с. 53-59], согласно которому $y_{r, k}=-\pi_{r, k}^{-1} \rho_{r, k+1}-\pi_{r, k}^{-1} \pi_{r, k+1}^{-1} \rho_{r, k+1}\left(\rho_{r, k+2}+\cdots+\rho_{r, l+1}\right)$. Так как $p_{r, k+1}=$ $\pi_{r, k+1}-\pi_{r, k}$, To

$$
y_{r, k}=\frac{1}{\pi_{r, k+1}} \sum_{i=k+2}^{l+1} \rho_{r, i}-\frac{1}{\pi_{r, k}} \sum_{i=k+1}^{l+1} \rho_{r, k}, \quad k=r, r+1, \ldots, l
$$

Мы придерживаемся соглашения, согласно которому сумма величин равна нулю, если нижний индекс суммирования больше верхнего индекса суммирования. По формуле (2) математическое ожидание случайной величины $n \rho_{r, k}^{2} / p_{r, k}$ равно $q_{r, k}=1-p_{r, k}$. Их сумма $q_{r, r}+\cdots+q_{r, l+1}$ равна $l-r+1$. Вычитая из каждого члена равенства $(20)$ одно и то же число $l-r+1$, получим

$$
\begin{gathered}
\sum_{k=r}^{l+1}\left(\frac{n}{p_{r, k}}\left(\frac{\nu_{r, k}}{n}-p_{r, k}\right)^{2}-q_{r, k}\right)=\sum_{k=r}^{l+1}\left(\frac{n}{p_{r, k}} \rho_{r, k}^{2}-q_{r, k}\right) \\
=\sum_{k=r}^{l}\left(\frac{n \pi_{r, k} \pi_{r, k+1}}{p_{r, k+1}} y_{r, k}^{2}-1\right)=\sum_{k=r}^{l} u_{r, k}
\end{gathered}
$$


где

$$
\begin{aligned}
u_{r, k} & =\frac{n \pi_{r, k} \pi_{r, k+1}}{p_{r, k+1}} y_{r, k}^{2}-1 \\
& =\frac{n \pi_{r, k} \pi_{r, k+1}}{p_{r, k+1}}\left(\frac{1}{\pi_{r, k+1}} \sum_{i=k+2}^{l+1} \rho_{r, i}-\frac{1}{\pi_{r, k}} \sum_{i=k+1}^{l+1} \rho_{r, i}\right)^{2}-1 .
\end{aligned}
$$

Лемма 1. Если величины $s$ u $p_{k}, k=1, \ldots, s$, зависят от $n$ maким образом, что $s \rightarrow \infty$ при $n \rightarrow \infty$ в выполнено условие (7) $c$ $W=\{1,2, \ldots, s\}, m o$

$$
\mathbf{E} u_{r, k}=0, \quad k=r, \ldots, l, \quad \mathbf{E} u_{r, l}^{2}=2+C_{1} \frac{1}{n p_{0}}
$$

где $p_{0}=p_{0}(\{1,2, \ldots, s\}) u C_{1}=C_{1}\left(r, l, n, p_{\tau}, \ldots, p_{l}\right)-\oint$ ункия, ограниченная по модулю нехоторой положительной постоянной $C$.

Д ок а з т ель с т о. См. лемму 3 в [2].

Определим величину $z_{h, r, k}$ посредством соотношения

$$
h=n\left(1-\pi_{r, k+1}\right)+z_{h, r, k} \sqrt{n \pi_{r, k+1}\left(1-\pi_{r, k+1}\right)}
$$

Лемма 2. Если выполнено условие

$$
\nu_{r, k+2}+\nu_{r, k+3}+\cdots+\nu_{r, l+1}=h
$$

mo

$$
\mathbf{E}_{h} u_{r, k}=-\frac{z_{h, r, k} \sqrt{1-\pi_{r, k+1}}}{\sqrt{n \pi_{r, k+1}}}, \quad k=r, r+1, \ldots, l-1,
$$

где символ $\mathbf{E}_{h}$ обозначает условное математическое ожидание при условии (23).

Д ок а за т ел в с т в о. См. лемму 4 в [2].

Лемма 3. Если величины $s$ u $p_{k}, k=1,2, \ldots, s$, зависят от $n$ таким образом, ито $s \rightarrow \infty$ при $n \rightarrow \infty$ в выполнено условие (7) $c$ $W=\{1,2, \ldots, s\}$, mo

$$
\begin{aligned}
\mathbf{E}_{h} u_{r, k}^{2}= & 2+C_{2} \mathbf{E}_{h} u_{r, k}+C_{3}\left(\mathbf{E}_{h} u_{r, k}\right)^{2}+C_{4} \frac{1}{n p_{0}} \\
\mathbf{E}_{h} u_{r, k}^{4}= & 60+C_{5} \mathbf{E}_{h} u_{r, k}+C_{6}\left(\mathbf{E}_{h} u_{r, k}\right)^{2}+C_{7}\left(\mathbf{E}_{h} u_{r, k}\right)^{3} \\
& +C_{8}\left(\mathbf{E}_{h} u_{r, k}\right)^{4}+C_{9} \frac{1}{n p_{0}}
\end{aligned}
$$

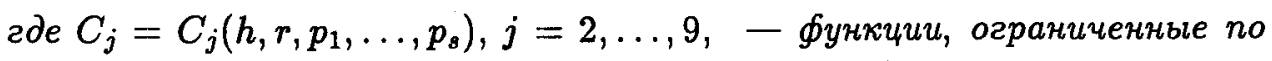
модулю некоторой положительной постоянной $D$. 
Д ок а з т ел в с в о. См. лемму 5 в [2]. Заметим попутно, что в [2] число 60 ошибочно стоит сомножителем при $C_{5}$.

Мы завершим введение обсуждением одного интересного свойства полиномиального распределения. Оно будет использовано при доказательстве теоремы 3. При доказательстве теоремы Туманяна [2] используется частный случай этого свойства как само собой разумеющееся, несмотря на то, что оно играет ключевую роль. Нам не удалось найти источник, где бы это свойство было сформулировано. Весьма вероятно, что сведения о нем составляют часть математического фольклора.

Лемма 4. Для любых челых чисел $h, l, t, 0 \leqslant h \leqslant n, 0 \leqslant l<t<s$, случайная величина $\nu_{t}$ и случайный вектор $\bar{\mu}=\left(\nu_{1}, \ldots, \nu_{l}, \nu_{t+1}, \ldots, \nu_{s}\right)$ $\left(\bar{\mu}=\left(\nu_{t+1}, \ldots, \nu_{s}\right)\right.$, если $l=0 u t=1, u \bar{\mu}=\left(\nu_{1}, \ldots, \nu_{s-1}\right)$, еслu $l=s-1$ $u t=s$ ) условно кезависимы при условии, ито $\nu_{1}+\cdots+\nu_{l}+\nu_{t+1}+\cdots+$ $\nu_{s}=h$.

Д ок азатель с т о. Утверждение справедливо при $l=0$ и $t=1$, а также при $l=s-1$ и $t=s$, так как в этих случаях случайные величины $\nu_{1}$ и $\nu_{s}$ почти всюду постоянны. Рассмотрим оставшиеся случаи. Обозначим $H=\nu_{1}+\cdots+\nu_{l}+\nu_{t+1}+\cdots+\nu_{s}, p_{l}^{(t)}=p_{1}+\cdots+p_{l}+p_{t}+\cdots+p_{s}$. Мы опускаем простые вычисления, согласно которым

$$
\begin{aligned}
& \mathbf{P}\left\{\nu_{1}=m_{1}, \ldots, \nu_{l}=m_{l}, \nu_{t}=m_{t}, \ldots, \nu_{s}=m_{s} \mid H=h\right\} \\
& =\frac{h !(n-h) !}{m_{1} ! \cdots m_{l} ! m_{t} ! \cdots m_{s} !\left(n-h-m_{t}\right) !} \\
& \quad \times \frac{p_{1}^{m_{1}} \cdots p_{l}^{m_{l}} p_{t}^{m_{t}} \cdots p_{s}^{m_{s}}\left(1-p_{l}^{(t)}\right)^{n-h-m_{t}}}{\left(p_{l}^{(t+1)}\right)^{h}\left(1-p_{l}^{(t+1)}\right)^{n-h}} \\
& \mathbf{P}\left\{\nu_{t}=m_{t} \mid H=h\right\}=\frac{(n-h) !}{m_{t} !\left(n-h-m_{t}\right) !} \frac{p_{t}^{m_{t}}\left(1-p_{l}^{(t)}\right)^{n-h-m_{t}}}{\left(1-p_{l}^{(t+1)}\right)^{n-h}}, \\
& \mathbf{P}\left\{\nu_{1}=m_{1}, \ldots, \nu_{l}=m_{l}, \nu_{t+1}=m_{t+1}, \ldots, \nu_{s}=m_{s} \mid H=h\right\} \\
& \quad=\frac{p_{1}^{m_{1}} \cdots p_{l}^{m_{l}} p_{t+1}^{m_{t+1}} \cdots p_{s}^{m s}}{\left(p_{l}^{(t+1)}\right)^{h}}
\end{aligned}
$$

и, следовательно,

$$
\begin{aligned}
\mathbf{P}\left\{\nu_{t}=m_{t} \mid H=h\right\} & \\
& \times \mathbf{P}\left\{\nu_{1}=m_{1}, \ldots, \nu_{l}=m_{l}, \nu_{t+1}=m_{t+1}, \ldots, \nu_{s}=m_{s} \mid H=h\right\} \\
= & \mathbf{P}\left\{\nu_{1}=m_{1}, \ldots, \nu_{l}=m_{l}, \nu_{t}=m_{t}, \ldots, \nu_{s}=m_{s} \mid H=h\right\} .
\end{aligned}
$$

Справедливость последнего равенства для любых допустимых значений случайных величин $\nu_{1}, \ldots, \nu_{l}, \nu_{t}, \ldots, \nu_{s}$ по определению означает условную независимость случайной величины $\nu_{t}$ и случайного вектора $\bar{\mu}$ при условии $H=h$. Лемма доказана. 
2. Многомерная предельная теорема. Пусть на вероятностном пространстве $(\Omega, \mathscr{F}, \mathbf{P})$ определен винеровский процесс $W(t), t \in[0,1]$, $W(0)=0$. Для каждого набора чисел $0=t_{0}<t_{1}<\cdots<t_{d} \leqslant 1$ его $d$-мерная функция распределения $\Phi_{t_{1}, \ldots, t_{d}}\left(x_{1}, \ldots, x_{d}\right)=\mathbf{P}\left\{W\left(t_{i}\right)<x_{i}, i=\right.$ $1, \ldots, d\}$ определяется своей характеристической функцией

$$
\begin{aligned}
f_{t_{1}, \ldots, t_{d}}\left(v_{1}, \ldots, v_{d}\right)= & \exp \left\{-\frac{1}{2} \sum_{j=1}^{d}\left(v_{j}+\cdots+v_{d}\right)^{2}\left(t_{j}-t_{j-1}\right)\right\}, \\
& \left(v_{1}, v_{2}, \ldots, v_{d}\right) \in \mathbf{R}^{d}
\end{aligned}
$$

Теорема 3. Предположим, что величины $s$ u $p_{k}, k=1,2, \ldots, s, u$ множество $W \subset\{1,2, \ldots, s\}$ зависят от $n$ таким образом, ито $s \rightarrow \infty$ при $n \rightarrow \infty$ и выполнены условия (6)-(8). Пусть $d-$ натуральное число, $t_{j} u\left\{s_{n}^{(j)}\right\}_{n \geqslant 1}, j=1,2, \ldots, d$, - положительные числа и неограниченные последовптельности натуральных чисел, удовлетворяющие условиям $0=t_{0}<t_{1}<\cdots<t_{d} \leqslant 1, s_{n}^{(j)} \leqslant s u s_{n}^{(j)} / s \rightarrow t_{j} n p u n \rightarrow \infty$. Тогда для любого вектора $\left(x_{1}, x_{2}, \ldots, x_{d}\right) \in \mathbf{R}^{d}$

$$
\begin{aligned}
& \lim _{n \rightarrow \infty} \mathbf{P}\left\{\frac{\chi_{n}^{2}\left(1, s_{n}^{(j)}\right)-\mathbf{E} \chi_{n}^{2}\left(1, s_{n}^{(j)}\right)}{\sqrt{\mathbf{D} \chi_{n}^{2}}}<x_{j}, j=1, \ldots, d\right\} \\
& =\Phi_{t_{1}, \ldots, t_{d}}\left(x_{1}, \ldots, x_{d}\right),
\end{aligned}
$$

ede $\chi_{n}^{2}\left(1, s_{n}^{(j)}\right)=\chi_{n}^{2}\left(W^{(j)}\right), W^{(j)}=\left\{1,2, \ldots, s_{n}^{(j)}\right\}$.

Д оказате льст в о. Обозначим $W_{j}=W \cap\left\{1,2, \ldots, s_{n}^{(j)}\right\}$, $\bar{W}_{j}=\bar{W} \cap\left\{1,2, \ldots, s_{n}^{(j)}\right\}$. Запишем числитель дроби, стоящей под знаком вероятности в (24), в следующем виде:

$$
\chi_{n}^{2}\left(1, s_{n}^{(j)}\right)-\mathbf{E} \chi_{n}^{2}\left(1, s_{n}^{(j)}\right)=\chi_{n}^{2}\left(W_{j}\right)-\mathbf{E} \chi_{n}^{2}\left(W_{j}\right)+\chi_{n}^{2}\left(\bar{W}_{j}\right)-\mathbf{E} \chi_{n}^{2}\left(\bar{W}_{j}\right)
$$

Точно так же, как (14), мы докажем, что

$$
\lim _{n \rightarrow \infty} \mathbf{P}\left\{\left|\frac{\chi_{n}^{2}\left(\bar{W}_{j}\right)-N\left(\bar{W}_{j}\right)}{\sqrt{2 s}}\right|>\varepsilon\right\}=0, \quad \varepsilon>0, j=1,2, \ldots, d
$$

Принимая во внимание равенство $\mathbf{E} \chi_{n}^{2}\left(\bar{W}_{j}\right)=N\left(\bar{W}_{j}\right)-\sum_{k \in \bar{W}_{j}} p_{k}$ и соотношение (15), мы видим, что (24) эквивалентно

$$
\lim _{n \rightarrow \infty} \mathbf{P}\left\{\frac{\chi_{n}^{2}\left(W_{j}\right)-\mathbf{E} \chi_{n}^{2}\left(W_{j}\right)}{\sqrt{2 s}}<x_{j}, j=1, \ldots, d\right\}=\Phi_{t_{1}, \ldots, t_{d}}\left(x_{1}, \ldots, x_{d}\right) .
$$

Пусть, например, $W=\{r, r+1, \ldots, l+1\}$. Общий случай сводится к этому частному случаю путем перенумерации слагаемых в сумме (1). C 
учетом (11) величину $\chi_{n}^{2}(W)$ можно записать в виде следующей суммы:

$$
\begin{aligned}
\chi_{n}^{2}(W)= & \sum_{k=r}^{l+1} \frac{n}{p_{r, k}}\left(\frac{\nu_{r, k}}{n}-p_{r, k}\right)^{2}+\frac{n}{p_{l+1}}\left(\frac{\nu_{l+1}}{n}-p_{l+1}\right)^{2} \\
& -\frac{n}{p_{r, l+1}}\left(\frac{\nu_{r, l+1}}{n}-p_{r, l+1}\right)^{2}
\end{aligned}
$$

Два последних слагаемых, деленных на $\sqrt{2 s}$, сходятся по вероятности $к$ нулю при $n \rightarrow \infty$. Поэтому выражение $\chi_{n}^{2}\left(W_{j}\right)$ строится по слагаемым статистики Пирсона

$$
\sum_{k=r}^{l+1} \frac{n}{p_{r, k}}\left(\frac{\nu_{r, k}}{n}-p_{r, k}\right)^{2} .
$$

В силу (7) выполняется условие $n \min _{r \leqslant k \leqslant l+1} p_{r, k} \longrightarrow \infty$ при $n \rightarrow \infty$. Из сказанного следует, что доказательство теоремы сводится $\mathbf{K}$ доказательству теоремы, когда условие (7) выполняется для $W=\{1,2, \ldots, s\}$. Далее мы будем считать это условие выполненным.

В силу (15) нормирующий множитель $\sqrt{\mathrm{D} \chi_{n}^{2}}$ в $(24)$ можно заменить величиной $\sqrt{2 s}$. Чтобы доказать теорему, по известной теореме непрерывности достаточно доказать, что для любого вектора $\left(v_{1}, v_{2}, \ldots, v_{d}\right) \in$ $\mathbf{R}^{d}$ характеристическая функция

$$
\begin{aligned}
& f_{n}\left(v_{1}, v_{2}, \ldots, v_{d}\right) \\
& \quad=\mathbf{E} \exp \left\{\frac{\iota}{\sqrt{2 s}} \sum_{j=1}^{d} v_{j}\left(\chi_{n}^{2}\left(1, s_{n}^{(j)}\right)-\mathbf{E} \chi_{n}^{2}\left(1, s_{n}^{(j)}\right)\right)\right\}, \quad \iota=\sqrt{-1},
\end{aligned}
$$

сходится при $n \rightarrow \infty$ к функции $f_{t_{1}, \ldots, t_{d}}\left(v_{1}, v_{2}, \ldots, v_{d}\right)$.

Нам удобно сначала преобразовать характеристическую функцию $f_{n}$. Из свойств последовательностей $\left\{s_{n}^{(j)}\right\}_{n \geqslant 1}$ следует, что выполняются неравенства $s_{n}^{(1)}<s_{n}^{(2)}<\cdots<s_{n}^{(d)}$ для всех достаточно больших значений $n$, скажем, для $n>N$. Далее будем считать, что $n>N$. Обратимся к преобразованиям случайных величин (10) и (19). Пусть $s_{n}^{(j-1)}+1$ и $s_{n}^{(j)}$ играют роли соответственно $r$ и $l$. Положим $s_{n}^{(0)}=0$,

$$
\begin{array}{ll}
\bar{p}_{j, k}=p_{s_{n}^{(j-1)}+1, k}, \quad \bar{\pi}_{j, k}=\pi_{s_{n}^{(j-1)}+1, k} \\
\bar{\nu}_{j, k}=\nu_{s_{n}^{(j-1)}+1, k}, \quad \bar{\rho}_{j, k}=\rho_{s_{n}^{(j-1)}+1, k}, \quad k=s_{n}^{(j-1)}+1, \ldots, s_{n}^{(j)}+1, \\
\bar{u}_{j, k}=u_{s_{n}^{(j-1)}+1, k}, \quad k=s_{n}^{(j-1)}+1, \ldots, s_{n}^{(j)} .
\end{array}
$$

По формуле (22)

$$
\bar{u}_{j, k}=\frac{n \bar{\pi}_{j, k} \bar{\pi}_{j, k+1}}{\bar{p}_{j, k+1}}\left(\frac{1}{\bar{\pi}_{j, k+1}} \sum_{i=k+2}^{s_{n}^{(j)}+1} \bar{\rho}_{j, i}-\frac{1}{\bar{\pi}_{j, k}} \sum_{i=k+1}^{s_{n}^{(j)}+1} \bar{\rho}_{j, i}\right)^{2}-1 .
$$


Если сумма $H_{j, k+2}=\bar{\nu}_{j, k+2}+\bar{\nu}_{j, k+3}+\cdots+\bar{\nu}_{j, s_{n}^{(j)}+1}, s_{n}^{(j-1)} \leqslant k \leqslant s_{n}^{(j)}-1$, равна целому числу $h, 0 \leqslant h \leqslant n$, то случайная величина $\bar{u}_{j, k}$ является функцией только одной случайной величины $\bar{\nu}_{j, k+1}=\nu_{k+1}$, так как $\sum_{i=k+2}^{s_{n}^{(j)}+1} \bar{\rho}_{j, i}=h / n-1+\bar{\pi}_{j, k+1}, \sum_{i=k+1}^{s_{n}^{(j)}+1} \bar{\rho}_{j, i}=\bar{u}_{j, k+1} / n+h / n-1+\bar{\pi}_{j, k}$. Сумму $H_{j, k+2}$ можно записать также в следующем виде:

$$
\begin{aligned}
H_{j, k+2} & =\nu_{k+2}+\cdots+\nu_{s_{n}^{(j)}}+n-\left(\nu_{s_{n}^{(j-1)}+1}+\cdots+\nu_{s_{n}^{(j)}}\right) \\
& =\nu_{1}+\cdots+\nu_{s_{n}^{(j-1)}}+\nu_{k+2}+\cdots+\nu_{s},
\end{aligned}
$$

так как по определению полиномиального распределения выполняется равенство $\nu_{1}+\nu_{2}+\cdots+\nu_{s}=n$. Если $H_{j, k+2}=h$, то по лемме 4 случайная величина $\nu_{k+1}$ условно не зависит от случайного вектора $\left(\nu_{1}, \ldots, \nu_{s_{n}^{(j-1)}}, \nu_{k+2}, \ldots, \nu_{s}\right)$ и, следовательно, случайная величина $\bar{u}_{j, k}$ не зависит от случайных величин $\bar{u}_{j, i}, k+1 \leqslant i \leqslant s_{n}^{(j)}$, и $\bar{u}_{\mu, i}$, $s_{n}^{(\mu-1)}+1 \leqslant i \leqslant s_{n}^{(\mu)}, \mu=j+1, \ldots, d$, так как они строятся в виде функций от случайных величин $\nu_{k+2}, \nu_{k+3}, \ldots, \nu_{s}$.

Обозначим $A\left(v_{1}, \ldots, v_{d}\right)$ выражение, стоящее под знаком экспоненты в определении характеристической функции $f_{n}$, без учета мнимой единицы $\iota$ и знаменателя $\sqrt{2 s}$, и преобразуем его следующим образом:

$$
\begin{aligned}
A & \left(v_{1}, \ldots, v_{d}\right)=\sum_{j=1}^{d} v_{j}\left(\chi_{n}^{2}\left(1, s_{n}^{(j)}\right)-\mathbf{E} \chi_{n}^{2}\left(1, s_{n}^{(j)}\right)\right) \\
= & \sum_{j=1}^{d} v_{j} \sum_{i=1}^{s_{n}^{(j)}}\left(\frac{n}{p_{i}}\left(\frac{\nu_{i}}{n}-p_{i}\right)^{2}-q_{i}\right) \\
= & \sum_{j=1}^{d} w_{j} \sum_{i=s_{n}^{(j-1)}+1}^{s_{n}^{(j)}}\left(\frac{n}{p_{i}}\left(\frac{\nu_{i}}{n}-p_{i}\right)^{2}-q_{i}\right)\left(w_{j}=v_{j}+v_{j+1}+\cdots+v_{d}\right) \\
= & \sum_{j=1}^{d} w_{j} \sum_{i=s_{n}^{(j-1)}+1}^{s_{n}^{(j)}+1}\left(\frac{n}{\bar{p}_{j, i}} \bar{\rho}_{j, i}^{2}-\bar{q}_{j, i}\right) \\
& -\sum_{j=1}^{d} w_{j}\left(\frac{n}{\bar{p}_{j, s_{n}}^{(j)}+1} \bar{\rho}_{j, s_{n}^{(j)}+1}^{2}-\bar{q}_{j, s_{n}^{(j)}+1}\right) \\
= & \sum_{j=1}^{d} w_{j} \sum_{i=s_{n}^{(j-1)}+1}^{s_{n}^{(j)}} \bar{u}_{j, i}-\sum_{j=1}^{d} w_{j}\left(\frac{n}{\bar{p}_{j, s_{n}}^{(j)}+1} \bar{\rho}_{j, s_{n}^{(j)}+1}^{2}-\bar{q}_{j, s_{n}^{(j)}+1}\right) .
\end{aligned}
$$

В этих преобразованиях мы воспользовались равенствами (21). Покажем, что последней суммой можно пренебречь. Подсчет дисперсии по 
формуле (2) применительно к слагаемым последней суммы дает

$$
\begin{aligned}
& \mathbf{E}\left(\frac{n}{\bar{p}_{j, s_{n}^{(j)}+1}} \bar{\rho}_{j, s_{n}^{(j)}+1}^{2}-\bar{q}_{j, s_{n}^{(j)}+1}\right)^{2} \\
& \quad=2 \bar{q}_{j, s_{n}^{(j)}+1}^{2}+\frac{\bar{q}_{j, s_{n}^{(j)}+1}}{n \bar{p}_{j, s_{n}^{(j)}+1}}\left(1-6 \bar{p}_{j, s_{n}^{(j)}+1} \bar{q}_{j, s_{n}^{(j)}+1}\right)
\end{aligned}
$$

Обозначим $V_{n, j}$ левую часть этого равенства. В силу условия (7) $V_{n, j} / 2 s \rightarrow 0$ при $n \rightarrow \infty$, и, следовательно, по неравенству Чебышева,

$$
\begin{aligned}
& \lim _{n \rightarrow \infty} \mathbf{P}\left\{\left|\frac{n}{\bar{p}_{j, s_{n}^{(j)}+1}} \bar{\rho}_{j, s_{n}^{(j)}+1}^{2}-\bar{q}_{j, s_{n}^{(j)}+1}\right|>\varepsilon \sqrt{2 s}\right\}=0, \\
& \epsilon>0, j=1,2, \ldots, d \text {. }
\end{aligned}
$$

Таким образом, чтобы доказать теорему 2, достаточно убедиться, что

$$
\begin{aligned}
& \lim _{n \rightarrow \infty} \mathbf{E} \exp \left\{\frac{1}{\sqrt{2 s}} \sum_{j=1}^{d} w_{j} \sum_{i=s_{n}^{(j-1)}+1}^{s_{n}^{(j)}} \bar{u}_{j, i}\right\} \\
& =\exp \left\{-\frac{1}{2} \sum_{j=1}^{d} w_{j}^{2}\left(t_{j}-t_{j-1}\right)\right\} .
\end{aligned}
$$

Обозначим

$$
\begin{array}{rl}
B_{n, \mu} & =\exp \left\{\frac{\iota}{\sqrt{2 s}} \sum_{j=\mu+1}^{d} w_{j} \sum_{i=s_{n}^{(j-1)}+1}^{s_{n}^{(j)}} \bar{u}_{j, i}\right\}, \mu=0,1, \ldots, d-1, \\
B_{n, d} & =1 \\
A_{n, \mu, k} & =\exp \left\{\frac{\iota}{\sqrt{2 s}} w_{\mu}\left(\bar{u}_{\mu, k}+\bar{u}_{\mu, k+1}+\cdots+\bar{u}_{\left.\mu, s_{n}^{(\mu)}\right)}\right\}\right. \\
k & k=s_{n}^{(\mu-1)}+1, \ldots, s_{n}^{(\mu)}, \\
C_{n, \mu, k} & =\mathbf{E}\left(A_{n, \mu, k} B_{n, \mu}\right) .
\end{array}
$$

Заметим, что функция $\mathbf{E} B_{n, 0}$ совпадает с функцией, стоящей под знаком предела в (25). Покажем, что функции $C_{n, \mu, k}$ связаны некоторым рекуррентным соотношением. Выше мы отметили, что при условии $H_{\mu, k+2}=\bar{\nu}_{\mu, k+2}+\cdots+\bar{\nu}_{\mu, s_{n}^{(\mu)}+1}=h$ случайная величина $\bar{u}_{\mu, k}$, $s_{n}^{(\mu-1)}+1 \leqslant k \leqslant s_{n}^{(\mu)}-1$, условно не зависит от случайных величин $\bar{u}_{\mu, i}, k+1 \leqslant i \leqslant s_{n}^{(\mu)}$, и $\bar{u}_{j, i}, s_{n}^{(j-1)} \leqslant i \leqslant s_{n}^{(j)}, j=\mu+1, \ldots, d$. Поэтому случайные величины $\bar{u}_{\mu, k}$ प $A_{n, \mu, k+1} B_{n, \mu}$ являются условно независимыми 
при условии $H_{\mu, k+2}=h$. По формуле полной вероятности

$$
\begin{aligned}
& C_{n, \mu, k}=\sum_{h=0}^{n} \mathbf{P}\left\{H_{\mu, k+2}=h\right\} \mathbf{E}_{h}\left(A_{n, \mu, k} B_{n, \mu}\right) \\
&= \sum_{h=0}^{n} \mathbf{P}\left\{H_{\mu, k+2}=h\right\} \mathbf{E}_{h} \exp \left\{\frac{\iota}{\sqrt{2 s}} w_{\mu} \bar{u}_{\mu, k}\right\} \mathbf{E}_{h}\left(A_{n, \mu, k+1} B_{n, \mu}\right), \\
& s_{n}^{(\mu-1)}+1 \leqslant k \leqslant s_{n}^{(\mu)}-1 .
\end{aligned}
$$

По формуле Тейлора

$$
\begin{gathered}
\mathbf{E}_{h} \exp \left\{\frac{\iota}{\sqrt{2 s}} w_{\mu} \bar{u}_{\mu, k}\right\}=1-\frac{w_{\mu}^{2}}{2 s}+\frac{\iota \dot{w}_{\mu}}{\sqrt{2 s}} \mathbf{E}_{h} \bar{u}_{\mu, k}+\frac{w_{\mu}^{2}}{4 s}\left(2-\mathbf{E}_{h} \bar{u}_{\mu, k}^{2}\right) \\
+\theta_{k} \frac{\iota^{3} w_{\mu}^{3}}{6(2 s)^{3 / 2}} \mathbf{E}_{h} \bar{u}_{\mu, k}^{3}, \quad\left|\theta_{k}\right|<1
\end{gathered}
$$

Подстановка этого выражения в предыдушую формулу приводит к следующему рекуррентному соотношению:

$$
C_{n, \mu, k}=\left(1-\frac{w_{\mu}^{2}}{2 s}\right) C_{n, \mu, k+1}+R_{n, \mu, k}
$$

где

$$
\begin{aligned}
R_{n, \mu, k} & =\sum_{h=0}^{n} \mathbf{P}\left\{H_{\mu, k+2}=h\right\} D_{h, \mu, k} \mathbf{E}_{h}\left(A_{n, \mu, k+1} B_{n, \mu}\right) \\
D_{h, \mu, k} & =\left(\frac{\iota w}{\sqrt{2 s}} \mathbf{E}_{h} \bar{u}_{\mu, k}+\frac{w_{\mu}^{2}}{4 s}\left(2-\mathbf{E}_{h} \bar{u}_{\mu, k}^{2}\right)+\theta_{k} \frac{\iota^{3} w_{\mu}^{3}}{6(2 s)^{3 / 2}} \mathbf{E}_{h} \bar{u}_{\mu, k}^{3}\right)
\end{aligned}
$$

Применим рекуррентное соотношение последовательно для $k=s_{n}^{(\mu-1)}+$ $1, \ldots, s_{n}^{(\mu)}-1$. Результатом будет

$$
\begin{aligned}
C_{n, \mu, s_{n}^{(\mu-1)}+1}= & C_{n, \mu, s_{n}^{(\mu)}}\left(1-\frac{w_{\mu}^{2}}{2 s}\right)^{s_{n}^{(\mu)}-s_{n}^{(\mu-1)}-1} \\
& +\sum_{k=s_{n}^{(\mu-1)}+1}^{s_{n}^{(\mu)}-1} R_{n, \mu, k}\left(1-\frac{w_{\mu}^{2}}{2 s}\right)^{k-s_{n}^{(\mu-1)}-1}
\end{aligned}
$$

Принимая во внимание равенство $C_{n, \mu, s_{n}^{(\mu-1)}+1}=\mathbf{E} B_{n, \mu-1}$, перепишем это соотношение в виде

$$
\begin{aligned}
\mathbf{E} B_{n, \mu-1}= & \mathbf{E} B_{n, \mu}\left(1-\frac{w_{\mu}^{2}}{2 s}\right)^{s_{n}^{(\mu)}-s_{n}^{(\mu-1)}-1} \\
& +L_{n, \mu}\left(1-\frac{w_{\mu}^{2}}{2 s}\right)^{s_{n}^{(\mu)}-s_{n}^{(\mu-1)}-1}+R_{n, \mu}, \quad \mu=1,2, \ldots, d,
\end{aligned}
$$


где

$$
L_{n, \mu}=C_{n, \mu, s_{n}^{(\mu)}}-\mathbf{E} B_{n, \mu}, \quad R_{n, \mu}=\sum_{k=s_{n}^{(\mu-1)}+1}^{s_{n}^{(\mu)}-1} R_{n, \mu, k}\left(1-\frac{w_{\mu}^{2}}{2 s}\right)^{k-s_{n}^{(\mu-1)}-1}
$$

Применим рекуррентное соотношение для функций $\mathbf{E} B_{n, \mu}$ последовательно для $\mu=1,2, \ldots, d$. В результате мы получим

$$
\begin{aligned}
\mathbf{E} B_{n, 0}= & \prod_{\mu=1}^{d}\left(1-\frac{w_{\mu}^{2}}{2 s}\right)^{s_{n}^{(\mu)}-s_{n}^{(\mu-1)}-1}+\sum_{\mu=1}^{d} L_{n, \mu} \prod_{j=1}^{\mu}\left(1-\frac{w_{j}^{2}}{2 s}\right)^{s_{n}^{(j)}-s_{n}^{(j-1)}-1} \\
& +\sum_{\mu=2}^{d} R_{n, \mu} \prod_{j=1}^{\mu-1}\left(1-\frac{w_{j}^{2}}{2 s}\right)^{s_{n}^{(j)}-s_{n}^{(j-1)}-1}+R_{n, 1}
\end{aligned}
$$

В силу свойств числовых последовательностей $\left\{s_{n}^{(\mu)}\right\}_{n \geqslant 1}$ выполняется соотношение

$$
\lim _{n \rightarrow \infty} \prod_{\mu=1}^{d}\left(1-\frac{w_{\mu}^{2}}{2 s}\right)^{s_{n}^{(\mu)}-s_{n}^{(\mu-1)}-1}=\exp \left\{-\frac{1}{2} \sum_{\mu=1}^{d} w_{\mu}^{2}\left(t_{\mu}-t_{\mu-1}\right)\right\}
$$

Привлекая неравенство $\left|e^{\iota \alpha}-1\right| \leqslant|\alpha|$, справедливое для любого вещественного числа $\alpha$, мы получим $\mathbf{E}\left|\exp \left\{\iota(2 s)^{-1 / 2} w_{\mu} \bar{u}_{\mu, s_{n}^{(\mu)}}\right\}-1\right| \leqslant$ $\left|w_{\mu}\right|(2 s)^{-1 / 2} \mathbf{E}\left|\bar{u}_{\mu, s_{n}^{(\mu)}}\right|, \mu=1,2, \ldots, d$. По неравенству Маркова для абсолютных моментов

$$
\frac{1}{\sqrt{2 s}} \mathbf{E}\left|\bar{u}_{\mu, s_{n}^{(\mu)}}\right| \leqslant \sqrt{\frac{1}{2 s} \mathbf{E} \bar{u}_{\mu, s_{n}^{(\mu)}}^{2}}=\sqrt{\frac{1}{2 s} \mathbf{E}{u_{s_{n}^{(\mu-1)}+1, s_{n}^{(\mu)}}^{2}}^{(\mu)}}
$$

В силу леммы 1 (напомним, что $s_{n}^{(\mu-1)}+1$ и $s_{n}^{(\mu)}$ играют роли $r$ и $l$ соответственно) правая часть этого неравенства стремится к нулю при $n \rightarrow \infty$, и, следовательно,

$$
\lim _{n \rightarrow \infty} \mathbf{E}\left|\exp \left\{\frac{\iota}{\sqrt{2 s}} w_{\mu} \bar{u}_{\mu, s_{n}^{(\mu)}}\right\}-1\right|=0, \quad \mu=1,2, \ldots, d .
$$

Из неравенства $\left|L_{n, \mu}\right|=\left|C_{n, \mu, s_{n}^{(\mu)}}-\mathbf{E} B_{n, \mu}\right| \leqslant \mathbf{E}\left|\exp \left\{\iota(2 s)^{-1 / 2} w_{\mu} \bar{u}_{\mu, s_{n}^{(\mu)}}\right\}-1\right|$ и (28) следует, что

$$
\lim _{n \rightarrow \infty} \sum_{\mu=1}^{d-1} L_{n, \mu} \prod_{j=1}^{\mu}\left(1-\frac{w_{j}^{2}}{2 s}\right)^{s_{n}^{(j)}-s_{n}^{(j-1)}-1}=0
$$


так как сомножители при $L_{\mu}$ в (29) положительны и ограничены сверху единицей для всех достаточно больших значений $n$, скажем, для $n>N$. Еслии $n>N$, то

$$
\begin{gathered}
\left|\sum_{\mu=2}^{d} R_{n, \mu} \prod_{j=1}^{\mu-1}\left(1-\frac{w_{j}^{2}}{2 s}\right)^{s_{n}^{(j)}-s_{n}^{(j-1)}-1}+R_{n, 1}\right| \\
\leqslant \sum_{\mu=1}^{d}\left|R_{n, \mu}\right| \leqslant \sum_{\mu=1}^{d} \sum_{k=s_{n}^{(\mu-1)}+1}^{s_{n}^{(\mu)}-1}\left|R_{n, \mu, k}\right| .
\end{gathered}
$$

Запишем $R_{n, \mu, k}$ в виде суммы трех слагаемых $\Sigma_{n, k, 1}, \Sigma_{n, k, 2}$ и $\Sigma_{n, k, 3}$, где

$$
\begin{aligned}
& \Sigma_{n, k, 1}=\frac{\iota w_{\mu}}{\sqrt{2 s}} \sum_{h=0}^{n} \mathbf{P}\left\{H_{\mu, k+2}=h\right\}\left(\mathbf{E}_{h} \bar{u}_{\mu, k}\right) \mathbf{E}_{h}\left(A_{n, \mu, k+1} B_{n, \mu}\right), \\
& \Sigma_{n, k, 2}=\frac{w_{\mu}^{2}}{4 s} \sum_{h=0}^{n} \mathbf{P}\left\{H_{\mu, k+2}=h\right\}\left(2-\mathbf{E}_{h} \bar{u}_{\mu, k}^{2}\right) \mathbf{E}_{h}\left(A_{n, \mu, k+1} B_{n, \mu}\right), \\
& \Sigma_{n, k, 3}=\theta_{k} \frac{\iota^{3} w_{\mu}^{3}}{6(2 s)^{3 / 2}} \sum_{h=0}^{n} \mathbf{P}\left\{H_{\mu, k+2}=h\right\}\left(\mathbf{E}_{h} \bar{u}_{\mu, k}^{3}\right) \mathbf{E}_{h}\left(A_{n, \mu, k+1} B_{n, \mu}\right) .
\end{aligned}
$$

Оценим каждую из этих трех величин. Заметим, что $\left|\mathbf{E}_{h}\left(A_{n, \mu, k+1} B_{n, \mu}\right)\right|$ $\leqslant 1$. По лемме 2

$$
\begin{aligned}
\left|\Sigma_{n, k, 1}\right| & \leqslant \frac{|w|}{\sqrt{2 s}} \sum_{h=0}^{n} \mathbf{P}\left\{H_{\mu, k+2}=h\right\}\left|\mathbf{E}_{h} \bar{u}_{\mu, k}\right| \\
& =\frac{|w|}{\sqrt{2 s}} \sum_{h=0}^{n} \mathbf{P}\left\{H_{\mu, k+2}=h\right\}\left|\mathbf{E}_{h} u_{s_{n}^{(\mu-1)}, k}\right| \\
& =\frac{|w|}{\sqrt{2 s}} \frac{\sqrt{1-\pi_{s_{n}^{(\mu-1)}+1, k+1}}}{\sqrt{n \pi_{s_{n}^{(\mu-1)}+1, k+1}}} \sum_{h=0}^{n} \mathbf{P}\left\{H_{\mu, k+2}=h\right\}\left|z_{h, s_{n}^{(\mu-1)}+1, k}\right| .
\end{aligned}
$$

Привлекая неравенство Коши-Буняковского, мы получим

$$
\begin{aligned}
& \sum_{h=0}^{n} \mathbf{P}\left\{H_{\mu, k+2}=h\right\}\left|z_{h, s_{n}^{(\mu-1)}+1, k}\right| \\
& \quad=\sum_{h=0}^{n} \sqrt{\mathbf{P}\left\{H_{\mu, k+2}=h\right\}} \sqrt{\mathbf{P}\left\{H_{\mu, k+2}=h\right\}}\left|z_{h, s_{n}^{(\mu-1)}+1, k}\right| \\
& \quad \leqslant \sqrt{\sum_{h=0}^{n} \mathbf{P}\left\{H_{\mu, k+2}=h\right\}\left|z_{h, s_{n}^{(\mu-1)}+1, k}\right|^{2}} \\
& \quad=\sqrt{\sum_{h=0}^{n} \mathbf{P}\left\{H_{\mu, k+2}=h\right\} \frac{\left(h-n\left(1-\pi_{s_{n}^{(\mu-1)}+1, k+1}\right)\right)^{2}}{n \pi_{s_{n}^{(\mu-1)}+1, k+1}\left(1-\pi_{s_{n}^{(\mu-1)}+1, k+1}\right)}}=1 .
\end{aligned}
$$


Последнее равенство легко проверить, если принять во внимание, что случайная величина $H_{\mu, k+2}=\bar{\nu}_{\mu, k+2}+\cdots+\bar{\nu}_{\mu, s_{n}^{(\mu)}+1}$ имеет биномиальное распределение с параметрами $n$ и $1-\pi_{s_{n}^{(\mu-1)}+1, k+1}$. Таким образом, имеет место оценка

$$
\begin{aligned}
\left|\Sigma_{n, k, 1}\right| & \leqslant \frac{|w|}{\sqrt{2 s}} \frac{\sqrt{1-\pi_{s_{n}^{(\mu-1)}+1, k+1}}}{\sqrt{n \pi_{s_{n}^{(\mu-1)}+1, k+1}}} \\
& \leqslant \frac{|w|}{\sqrt{2 s}} \frac{1}{\sqrt{n p_{0}\left(k+1-s_{n}^{(\mu-1)}\right)}}, \quad k=s_{n}^{(\mu-1)}+1, \ldots, s_{n}^{(\mu)} .
\end{aligned}
$$

Оценим $\Sigma_{n, k, 2}$. По лемме 3

$$
\begin{aligned}
\left|2-\mathbf{E}_{h} \bar{u}_{\mu, k}^{2}\right| & =\left|2-\mathbf{E}_{h}{u_{s_{n}^{(\mu-1)}+1, k}^{2}}^{2}\right| \\
& \leqslant D\left(\left|\mathbf{E}_{h} u_{s_{n}^{(\mu-1)}+1, k}\right|+\left(\mathbf{E}_{h} u_{s_{n}^{(\mu-1)}+1, k}\right)^{2}+\frac{1}{n p_{0}}\right)
\end{aligned}
$$

и, следовательно,

$$
\begin{aligned}
\left|\Sigma_{n, k, 2}\right| \leqslant D \frac{w_{\mu}^{2}}{4 s}\left(\sum_{h=0}^{n} \mathbf{P}\left\{H_{\mu, k+2}=h\right\} \mathbf{E}_{h}\left|u_{s_{n}^{(\mu-1)}+1, k}\right|\right. \\
\left.+\sum_{h=0}^{n} \mathbf{P}\left\{H_{\mu, k+2}=h\right\}\left(\mathbf{E}_{h} u_{s_{n}^{(\mu-1)}+1, k}\right)^{2}+\frac{1}{n p_{0}}\right) .
\end{aligned}
$$

При оценке величины $\Sigma_{n, k, 1}$ было установлено, что

$$
\begin{aligned}
& \sum_{h=0}^{n} \mathbf{P}\left\{H_{\mu, k+2}=h\right\} \mathbf{E}_{h}\left|u_{s_{n}^{(\mu-1)}+1, k}\right| \leqslant \frac{\sqrt{1-\pi_{s_{n}^{(\mu-1)}+1, k+1}}}{\sqrt{n \pi_{s_{n}^{(\mu-1)}+1, k+1}}} \\
& \sum_{h=0}^{n} \mathbf{P}\left\{H_{\mu, k+2}=h\right\}\left(\mathbf{E}_{h} u_{s_{n}^{(\mu-1)}+1, k}\right)^{2} \\
& =\frac{1-\pi_{s_{n}^{(\mu-1)}+1, k+1}}{n \pi_{s_{n}^{(\mu-1)}+1, k+1}} \sum_{h=0}^{n} \mathbf{P}\left\{H_{\mu, k+2}=h\right\} z_{h, s_{n}^{(\mu-1)}+1, k}^{2} \\
& =\frac{1-\pi_{s_{n}^{(\mu-1)}+1, k+1}}{n \pi_{s_{n}^{(\mu-1)}+1, k+1}}
\end{aligned}
$$

и, следовательно,

$$
\begin{aligned}
\left|\Sigma_{n, k, 2}\right| & \leqslant D \frac{w_{\mu}^{2}}{4 s}\left(\frac{\sqrt{1-\pi_{s_{n}^{(\mu-1)}+1, k+1}}}{\sqrt{n \pi_{s_{n}^{(\mu-1)}+1, k+1}}}+\frac{1-\pi_{s_{n}^{(\mu-1)}+1, k+1}}{n \pi_{s_{n}^{(\mu-1)}+1, k+1}}+\frac{1}{n p_{0}}\right) \\
& \leqslant D \frac{w_{\mu}^{2}}{4 s}\left(\frac{1}{\sqrt{n p_{0}}}+\frac{2}{n p_{0}}\right), \quad k=s_{n}^{(\mu-1)}+1, \ldots, s_{n}^{(\mu)} .
\end{aligned}
$$


Оценим $\Sigma_{n, k, 3}$. По неравенству Маркова для абсолютных моментов

$$
\begin{aligned}
\mathbf{E}_{h}\left|u_{s_{n}^{(\mu-1)}+1, k}\right| & =\mathbf{E}_{h}\left|\bar{u}_{\mu, k}\right| \leqslant\left(\mathbf{E}_{h}\left|\bar{u}_{\mu, k}\right|^{3}\right)^{1 / 3} \leqslant\left(\mathbf{E}_{h} \bar{u}_{\mu, k}^{4}\right)^{1 / 4} \\
& =\left(\mathbf{E}_{h} u_{s_{n}^{(\mu-1)}+1, k}^{4}\right)^{1 / 4} .
\end{aligned}
$$

В силу леммы 3 выполняются неравенства

$$
\begin{array}{ll}
\mathbf{E}_{h} u_{s_{n}^{(\mu-1)}+1, k}^{4} \leqslant C_{10}, & \text { если }\left|\mathbf{E}_{h} u_{s_{n}^{(\mu-1)}+1, k}\right| \leqslant 1, \\
\mathbf{E}_{h} u_{s_{n}^{(\mu-1)}+1, k}^{4} \leqslant C_{11}\left(\mathbf{E}_{h} u_{s_{n}^{(\mu-1)}+1, k}\right)^{4}, & \text { если }\left|\mathbf{E}_{h} u_{s_{n}^{(\mu-1)}+1, k}\right|>1,
\end{array}
$$

где $C_{10}$ и $C_{11}$ - некоторые положительные постоянные. В силу этих неравенств

$$
\begin{aligned}
& \left|\Sigma_{n, k, 3}\right| \leqslant \frac{\left|w_{\nu}\right|^{3}}{6(2 s)^{3 / 2}} \sum_{h=0}^{n} \mathbf{P}\left\{H_{\mu, k+2}=h\right\}\left|\mathbf{E}_{h} \bar{u}_{\mu, k}\right|^{3} \\
& \leqslant \frac{\left|w_{\nu}\right|^{3}}{6(2 s)^{3 / 2}} \sum_{h=0}^{n} \mathbf{P}\left\{H_{\mu, k+2}=h\right\}\left(\mathbf{E}_{h} u_{s_{n}^{(\mu-1)}+1, k}^{4}\right)^{3 / 4} \\
& =\frac{\left|w_{\nu}\right|^{3}}{6(2 s)^{3 / 2}}\left(\sum_{h=0}^{n} \mathbf{P}\left\{H_{\mu, k+2}=h\right\}\left(\mathbf{E}_{h}{u_{s_{n}^{(\mu-1)}+1, k}^{4}}^{4}\right)^{3 / 4}\right. \\
& \left.+\sum_{h=0}^{n} \mathbf{P}\left\{H_{\mu, k+2}=h\right\}\left(\mathbf{E}_{h} u_{s_{n}^{(\mu-1)}+1, k}^{4}\right)^{3 / 4}\right) \\
& \leqslant \frac{\left|w_{\mu}\right|^{3}}{6(2 s)^{3 / 2}} C_{10}^{3 / 4}+\frac{\left|w_{\mu}\right|^{3}}{6(2 s)^{3 / 2}} \\
& \times C_{11}^{3 / 4} \sum_{h=0}^{n} \mathbf{P}\left\{H_{\mu, k+2}=h\right\}\left(\mathbf{E}_{h} u_{s_{n}^{(\mu-1)}+1, k}\right)^{4},
\end{aligned}
$$

где символы $\Sigma^{\prime}$ и $\sum^{\prime \prime}$ означают, что суммирование распространяется на слагаемые, удовлетворяющие соответственно условиям $\left|\mathbf{E}_{h} u_{s_{n}^{(\mu-1)}+1, k}\right| \leqslant 1$ и $\left|\mathbf{E}_{h} u_{s_{n}^{(\mu-1)}+1, k}\right|>1$. По лемме 2

$$
\begin{aligned}
& \sum_{h=0}^{n} \mathbf{P}\left\{H_{\mu, k+2}=h\right\}\left(\mathbf{E}_{h} u_{s_{n}^{(\mu-1)}+1, k}\right)^{4} \\
& \quad=\frac{\left(1-\pi_{s_{n}^{(\mu-1)}+1, k+1}\right)^{2}}{n^{2} \pi_{s_{n}^{(\mu-1)}+1, k+1}^{2}} \sum_{h=0}^{n} \mathbf{P}\left\{H_{\mu, k+2}=h\right\} z_{h, s_{n}^{(\mu-1)}+1, k}^{4} \\
& \quad=\frac{\left(1-\pi_{s_{n}^{(\mu-1)}+1, k+1}\right)^{2}}{n^{2} \pi_{s_{n}^{(\mu-1)}+1, k+1}^{2}} \\
& \times \sum_{h=0}^{n} \mathbf{P}\left\{H_{\mu, k+2}=h\right\} \frac{\left(h-n\left(1-\pi_{s_{n}^{(\mu-1)}+1, k+1}\right)\right)^{4}}{n^{2} \pi_{s_{n}^{2(\mu-1)}+1, k+1}^{\left(1-\pi_{s_{n}^{(\mu-1)}+1, k+1}\right)^{2}}}
\end{aligned}
$$


Выше упоминалось, что случайная величина $H_{\mu, k+2}$ имеет биномиальное распределение с параметрами $n$ и $1-\pi_{s_{n}^{(\mu-1)}+1, k+1}$. Стандартные вычисления приводят к равенству

$$
\begin{aligned}
\sum_{h=0}^{n} \mathbf{P} & \left\{H_{\mu, k+2}=h\right\}\left(h-n\left(1-\pi_{s_{n}^{(\mu-1)}+1, k+1}\right)\right)^{4} \\
= & 3 n^{2}\left(1-\pi_{s_{n}^{(\mu-1)}+1, k+1}\right)^{2} \pi_{s_{n}^{(\mu-1)}+1, k+1}^{2}+n\left(1-\pi_{s_{n}^{(\mu-1)}+1, k+1}\right) \\
& \times \pi_{s_{n}^{(\mu-1)}+1, k+1}\left(1-6\left(1-\pi_{s_{n}^{(\mu-1)}+1, k+1}\right) \pi_{s_{n}^{(\mu-1)}+1, k+1}\right),
\end{aligned}
$$

и, следовательно,

$$
\begin{gathered}
\sum_{h=0}^{n} \mathbf{P}\left\{H_{\mu, k+2}=h\right\}\left(\mathbf{E}_{h} u_{s_{n}^{(\mu-1)}+1, k}\right)^{4} \\
=\frac{\left(1-\pi_{s_{n}^{(\mu-1)}+1, k+1}\right)^{2}}{n^{2} \pi^{2}(3-1)+1, k+1}\left(3+\frac{1-6\left(1-\pi_{s_{n}^{(\mu-1)}+1, k+1}\right) \pi_{s_{n}^{(\mu-1)}+1, k+1}}{n \pi_{s_{n}^{(\mu-1)}+1, k+1}\left(1-\pi_{s_{n}^{(\mu-1)}+1, k+1}\right)}\right) \\
\leqslant \frac{1}{n^{2} p_{0}^{2}}\left(3+\frac{7}{n p_{0}}\right) \leqslant \sup _{n \geqslant 1} \frac{1}{n^{2} p_{0}^{2}}\left(3+\frac{7}{n p_{0}}\right) \stackrel{\text { def }}{=} C_{12} \\
\left|\Sigma_{n, k, 3}\right| \leqslant \frac{\left|w_{\mu}\right|^{3}}{6(2 s)^{3 / 2}} E, \quad E \stackrel{\text { def }}{=}\left(C_{10}^{3 / 4}+C_{11}^{3 / 4} C_{12}\right) \\
k=s_{n}^{(\mu-1)}+1, \ldots, s_{n}^{(\mu)}
\end{gathered}
$$

Собирая оценки (31), (32) и (33), мы получим

$$
\begin{aligned}
\left|R_{n, \mu, k}\right| \leqslant & \left|\Sigma_{n, k, 1}\right|+\left|\Sigma_{n, k, 2}\right|+\left|\Sigma_{n, k, 3}\right| \leqslant \frac{\left|w_{\mu}\right|}{\sqrt{2 s}} \frac{1}{\sqrt{n p_{0}}} \frac{1}{\sqrt{k+1-s_{n}^{(\mu-1)}}} \\
& +\frac{w_{\mu}^{2}}{4 s} D\left(\frac{1}{\sqrt{n p_{0}}}+\frac{2}{n p_{0}}\right)+\frac{\left|w_{\mu}\right|^{3}}{6(2 s)^{3 / 2}} E, \\
\left|R_{n, \mu}\right| \leqslant & \sum_{k=s_{n}^{(\mu-1)}+1}^{s_{n}^{(\mu)}}\left|R_{n, \mu, k}\right| \leqslant \frac{\left|w_{\mu}\right|}{\sqrt{2 s}} \frac{1}{\sqrt{n p_{0}}} \sum_{k=s_{n}^{(\mu-1)}+1}^{s_{n}^{(\mu)}} \frac{1}{\sqrt{k+1-s_{n}^{(\mu-1)}}} \\
& +\frac{s_{n}^{(\mu)}-s_{n}^{(\mu-1)}}{4 s} w_{\mu}^{2} D\left(\frac{1}{\sqrt{n p_{0}}}+\frac{2}{n p_{0}}\right)+\frac{s_{n}^{(\mu)}-s_{n}^{(\mu-1)}}{6(2 s)^{3 / 2}}\left|w_{\mu}\right|^{3} E .
\end{aligned}
$$

Нетрудно видеть, что

$$
\sum_{k=s_{n}^{(\mu-1)}+1}^{s_{n}^{(\mu)}} \frac{1}{\sqrt{k+1-s_{n}^{(\mu-1)}}} \leqslant \int_{s_{n}^{(\mu-1)}+1}^{s_{n}^{(\mu)}} \frac{d x}{\sqrt{x-s_{n}^{(\mu-1)}}} \leqslant \sqrt{s_{n}^{(\mu)}} .
$$


Привлекая условие теоремы $s_{n}^{(\mu)} \leqslant s$, мы приходим к оценке

$$
\left|R_{n, \mu}\right| \leqslant\left|w_{\mu}\right| \frac{1}{\sqrt{n p_{0}}}+w_{\mu}^{2} D\left(\frac{1}{\sqrt{n p_{0}}}+\frac{1}{n p_{0}}\right)+\frac{1}{s^{1 / 2}}\left|w_{\mu}\right|^{3} E,
$$

и, следовательно, в силу условия (7),

$$
\lim _{n \rightarrow \infty}\left(\sum_{\mu=2}^{d} R_{n, \mu} \prod_{j=1}^{\mu-1}\left(1-\frac{w_{j}^{2}}{2 s}\right)^{s_{n}^{(j)}-s_{n}^{(j-1)}-1}+R_{n, 1}\right)=0
$$

так как сомножители при $R_{n, \mu}, \mu=1,2, \ldots, d$, ограничены по модулю единицей для всех достаточно больших $\boldsymbol{n}$. Из последнего соотношения, (26), (27) и (29) следует (25). Теорема доказана.

3. Неравенство для максимальной суммы. Статистика Пирсона представляет собой сумму зависимых случайных величин. В следующем пункте нам потребуется оценка вероятности того, что максимальная частичная центрированная сумма превзойдет данный уровень. Необходимое неравенство будет получено как следствие одной теоремы, принадлежащей П. Биллингсли. Приведем ее формулировку.

Пусть $X_{1}, X_{2}, \ldots, X_{m}$ - случайные величины, которые не обязаны быть независимыми. Положим $S_{0}=0, S_{k}=X_{1}+X_{2}+\cdots+X_{k}, k=$ $1,2, \ldots, m$.

Теорема Биллингсли. Предположим, что существуют положительные постоянные $u_{1}, u_{2}, \ldots, u_{m}$ mахие, что $\mathbf{E}\left|S_{j}-S_{i}\right|^{\gamma} \leqslant$ $\left(\sum_{l=i+1}^{j} u_{l}\right)^{\alpha}, 0 \leqslant i<j \leqslant m$, для нехоторых $\gamma>0 u \alpha>1$. Тогда для всех положительных $\lambda$

$$
\mathbf{P}\left\{\max _{1 \leqslant k \leqslant m}\left|S_{k}\right| \geqslant \lambda\right\} \leqslant \frac{K_{\gamma, \alpha}}{\lambda^{\gamma}}\left(\sum_{l=1}^{m} u_{l}\right)^{\alpha},
$$

где постоянная $K_{\gamma, \alpha}$ зависит только от $\alpha u \gamma$.

Д ок а з а т л в с т во. См. $[9$, с. 134].

Лемма 5. Пусть величины $s$ $u p_{k}, k=1,2, \ldots, s$, зависят от $n$ таким образом, ито

$$
\inf _{n}\left\{n \min _{1 \leqslant k \leqslant s} p_{k}\right\}=\alpha>0, \quad \sup _{n}\left\{\frac{s}{n}\right\}=\beta<\infty .
$$

Тогда для любых челых чисел $r$ u $m, 1 \leqslant r<m \leqslant s, u$ любого числа $\lambda>0$

$$
\mathbf{P}\left\{\max _{r \leqslant l \leqslant m}\left|\sum_{k=r}^{l}\left(\frac{n}{p_{k}}\left(\frac{\nu_{k}}{n}-p_{k}\right)^{2}-q_{k}\right)\right| \geqslant \lambda\right\} \leqslant \frac{C}{\lambda^{4}}(m+1-r)^{2},
$$

где $C$ - некоторая положительная постоянная. 
Д о к а з а т е л в с т в о. В [10] приведены вычисления ряда центральных моментов статистики Пирсона и, в частности, четвертого центрального момента

$$
\begin{aligned}
\mathbf{E}\left(\chi_{n}^{2}-\mathbf{E} \chi_{n}^{2}\right)^{4}= & 12(s-1)(s+3) \\
+ & 12\left[(s-31) R_{1}-\left(s^{3}+25 s^{2}+44 s-38\right)\right] n^{-1} \\
+ & {\left[112 R_{2}+3 R_{1}^{2}-2\left(3 s^{2}+118 s+658\right) R_{1}\right.} \\
& \left.+3\left(s^{4}+44 s^{3}+328 s^{2}+488 s-380\right)\right] n^{-2} \\
+ & {\left[R_{3}-4(s+28) R_{2}-3 R_{1}^{2}+4\left(3 s^{2}+56 s+236\right) R_{1}\right.} \\
& \left.-6\left(s^{4}+20 s^{3}+116 s^{2}+160 s-120\right)\right] n^{-3},
\end{aligned}
$$

где $R_{1}=\sum_{k=1}^{s} p_{k}^{-1}, R_{2}=\sum_{k=1}^{s} p_{k}^{-2}, R_{3}=\sum_{k=1}^{s} p_{k}^{-3}$. Из этого выражения следует, что

$$
\begin{gathered}
\mathbf{E}\left(\chi_{n}^{2}-\mathbf{E} \chi_{n}^{2}\right)^{4} \leqslant A s^{2}, \\
A=\sup _{n}\left\{s^{-2} \mathbf{E}\left(\chi_{n}^{2}-\mathbf{E} \chi_{n}^{2}\right)^{4}\right\} \\
<1224+1464 \beta+1119 \beta^{2}+\frac{236 \beta^{2}+944 \beta+384}{\alpha}+\frac{115}{\alpha^{2}}+\frac{1}{\alpha^{3}} .
\end{gathered}
$$

Обратимся к преобразованиям случайных величин (10). Математическое ожидание $k$-го слагаемого в сумме, стоящей слева в (11), равно $q_{r, k}=1-p_{r, k}$. Вычитая их сумму из обеих частей равенства (11), мы получим

$$
\begin{aligned}
\sum_{k=r}^{l+1}\left(\frac{n}{p_{r, k}}\left(\frac{\nu_{r, k}}{n}-p_{r, k}\right)^{2}-q_{r, k}\right)= & \chi_{n}^{2}(r, l)-\mathbf{E} \chi_{n}^{2}(r, l) \\
& +\frac{n}{p_{r, l+1}}\left(\frac{\nu_{r, l+1}}{n}-p_{r, l+1}\right)^{2}-q_{r, l+1}
\end{aligned}
$$

Применение элементарного неравенства $(a+b)^{4} \leqslant 16\left(|a|^{4}+|b|^{4}\right)$, справедливого для любых вешественных чисел, дает

$$
\begin{aligned}
& \mathbf{E}\left(\chi_{n}^{2}(r, l)-\mathbf{E} \chi_{n}^{2}(r, l)\right)^{4} \\
& \leqslant 16 \mathbf{E}\left(\left|\sum_{k=r}^{l+1}\left(\frac{n}{p_{r, k}}\left(\frac{\nu_{r, k}}{n}-p_{r, k}\right)^{2}-q_{r, k}\right)\right|^{4}\right. \\
& \left.+\left|\frac{n}{p_{r, l+1}}\left(\frac{\nu_{r, l+1}}{n}-p_{r, l+1}\right)^{2}-q_{r, l+1}\right|^{4}\right) .
\end{aligned}
$$


Оценим слагаемые в правой части (36). К первому из них можно применить неравенство (35), согласно которому

$$
\mathbf{E}\left|\sum_{k=r}^{l+1}\left(\frac{n}{p_{r, k}}\left(\frac{\nu_{r, k}}{n}-p_{r, k}\right)^{2}-q_{r, k}\right)\right|^{4} \leqslant A(l+2-r)^{2} \leqslant 4 A(l+1-r)^{2} .
$$

По поводу второго слагаемого заметим, что случайная величина $\nu_{r, l+1}=$ $n-\nu_{r}-\cdots-\nu_{l}$ имеет биномиальное распределение с параметрами $n$ и $p_{r, l+1}=1-p_{r}-\cdots-p_{l}$. Обозначим временно $p_{r, l+1}=p, q_{r, l+1}=1-p=q$, и воспользуемся вычислениями из [2], согласно которым

$$
\begin{aligned}
\mathbf{E}\left(\nu_{r, l+1}-n p_{r, l+1}\right)^{8}= & 105 n^{4} p^{4} q^{4}+70 n^{3} p^{3} q^{3}(7-34 p q) \\
& +7 n^{2} p^{2} q^{2}\left(17-308 p q+1044 p^{2} q^{2}\right) \\
& +n p q\left(1-126 p q+1680 p^{2} q^{2}-5040 p^{3} q^{3}\right)
\end{aligned}
$$

Из этого выражения следует, что

$$
\sup _{n} \mathbf{E}\left(\frac{n}{p_{r, l+1}}\left(\frac{\nu_{r, l+1}}{n}-p_{r, l+1}\right)^{2}\right)^{4}=B<105+\frac{490}{\alpha}+\frac{7427}{\alpha^{2}}+\frac{1681}{\alpha^{3}} .
$$

Привлекая еще раз упомянутое элементарное неравенство, мы получим

$$
\begin{aligned}
\mathbf{E} & \left|\frac{n}{p_{r, l+1}}\left(\frac{\nu_{r, l+1}}{n}-p_{r, l+1}\right)^{2}-q_{r, l+1}\right|^{4} \\
& \leqslant 16 \mathbf{E}\left(\left(\frac{n}{p_{r, l+1}}\left(\frac{\nu_{r, l+1}}{n}-p_{r, l+1}\right)^{2}\right)^{4}+q_{r, l+1}^{4}\right) \leqslant 16(B+1) .
\end{aligned}
$$

Замена слагаемых в правой части (36) их оценками дает

$$
\begin{aligned}
\mathbf{E}\left(\chi_{n}^{2}(r, l)-\mathbf{E} \chi_{n}^{2}(r, l)\right)^{4} & \leqslant 16\left(4 A(l+1-r)^{2}+16(B+1)\right) \\
& \leqslant(64 A+256 B+256)(l+1-r)^{2} .
\end{aligned}
$$

Теперь неравенство (34) является следствием теоремы Биллингсли, в которой следует положить $\alpha=2, \gamma=4$ и $u_{1}=u_{2}=\cdots=u_{m}=$ $\sqrt{64 A+256 B+256}$. В качестве постоянной $C$, фигурирующей в (34), можно взять $C=K_{4,2}(64 A+256 B+256)$, где $K_{4,2}$ - постоянная, о которой говорится в теореме Биллингсли. Лемма доказана.

4. Сходимость случайных ломаных. Обозначим

$$
\begin{gathered}
X_{n, k}=\frac{n}{p_{k}}\left(\frac{\nu_{k}}{n}-p_{k}\right)^{2}-q_{k}, \quad S_{n, k}=X_{n, 1}+X_{n, 2}+\cdots+X_{n, k} \\
t_{0}^{(n)}=0, \quad t_{k}^{(n)}=\frac{k}{s}, \quad k=1,2, \ldots, s .
\end{gathered}
$$


Определим случайную ломаную $X_{n}(t), t \in[0,1]$, с вершинами в точках $\left(t_{k}^{(n)}, S_{n, k} / \sqrt{2 s}\right)$. На отрезке $\left[t_{k-1}^{(n)}, t_{k}^{(n)}\right]$ она имеет вид

$$
X_{n}(t)=\frac{1}{\sqrt{2 s}}\left(S_{n, k-1}+\frac{t-t_{k}^{(n)}}{t_{k}^{(n)}-t_{k-1}^{(n)}} X_{n, k}\right) .
$$

Случайная ломаная представляет собой случайный процесс. Ero pacпределение $\mathbf{P}_{n}$ представляет собой вероятностную меру, определенную на борелевской сигма-алгебре подмножеств банахова пространства $C[0,1]$ непрерывньх функций $x(t), t \in[0,1]$, с нормой $\|x\|=\sup _{0 \leqslant t \leqslant 1}|x(t)|$. Известно, что на этой сигма-алгебре можно определить винеровскую меру $\mathbf{P}_{0}$. Пусть, как и в п. $2, W(t), t \in[0,1], W(0)=0$, обозначает винеровский процесс с распределением $\mathbf{P}_{0}$.

Теорема 4. Предположим, что величины $s$ u $p_{k}, k=1,2, \ldots, s, u$ множество $W \subset\{1,2, \ldots, s\}$ зависят от $n$ таким образом, ито $s \rightarrow \infty$ при $n \rightarrow \infty$ и выполнены условия (6)-(8). Тогда последовательность распределений $\left\{\mathbf{P}_{n}\right\}_{n \geqslant 1}$ слабо сходится $\kappa$ винеровской мере $\mathbf{P}_{0}$.

Д ок аз а т е л в с т о. В соответствии с теоремой Прохорова [11] достаточно доказать, что последовательность $\left\{\mathbf{P}_{n}\right\}_{n \geqslant 1}$ слабо компактна и конечномерные распределения случайного процесса $X_{n}(t), t \in[0,1]$, слабо сходятся к соответствующим конечномерным распределениям винеровского процесса $W(t), t \in[0,1]$. Установим сначала наличие свойства слабой компактности у последовательности $\left\{\mathbf{P}_{n}\right\}_{n \geqslant 1}$. Обозначим $S(A, \delta)=\{x \in C[0,1]:\|x-y\|<\delta, y \in A\}-\delta$-окрестность множества $A \subset C[0,1]$. Известно [11], что слабая компактность последовательности $\left\{\mathbf{P}_{n}\right\}_{n \geqslant 1}$ равносильна следующему условию: для любых $\varepsilon>0$ и $\delta>0$ найдется такое конечное множество $X(\varepsilon, \delta) \subset C[0,1]$, что $\mathbf{P}_{n}(S(X(\varepsilon, \delta), \delta))>1-\varepsilon$ для всех $n$ за исключением, быть может, конечного числа. Докажем это. Для этой цели разобьем отрезок $[0,1]$ на части: $\Delta_{0}^{r}=[0,1 / r], \Delta_{\nu}^{r}=(\nu / r,(\nu+1) / r], 1 \leqslant \nu \leqslant r-1$, где $r-$ натуральное число. Образуем множества функций из $C[0,1]$ :

$$
D_{\nu}^{r}=\left\{x: \max _{t \in \Delta_{\nu}^{r}}\left|x(t)-x\left(\frac{\nu}{r}\right)\right| \geqslant \delta\right\}, \quad 0 \leqslant \nu \leqslant r-1 .
$$

Обозначим $i_{\nu}=i_{n, \nu}$ и $j_{\nu}=j_{n, \nu}$ минимальные номера, удовлетворяющие условиям $\nu / r \leqslant t_{i_{\nu}}^{(n)}$ и $(\nu+1) / r \leqslant t_{j_{\nu}}^{(n)}$. В силу выбора $i_{\nu}$ и $j_{\nu}$ выполняется соотношение

$$
\lim _{n \rightarrow \infty} \frac{j_{\nu}-i_{\nu}}{s}=\frac{1}{r}
$$

Из определения случайной ломаной следует, что

$$
\left\{X_{n}(t) \in D_{\nu}^{r}\right\} \subset\left\{\max _{i_{\nu} \leqslant k \leqslant j_{\nu}}\left|\sum_{i=i_{\nu}}^{k} X_{n, i}\right| \geqslant \frac{\delta}{4} \sqrt{2 s}\right\} .
$$


Действительно, если, например, $t_{j_{\nu}-1}^{(n)}<t \leqslant(\nu+1) / r$, то

$$
\begin{aligned}
\sqrt{2 s}\left(X_{n}(t)-X_{n}\left(\frac{\nu}{r}\right)\right)= & S_{n, j_{\nu}-1}+\frac{t-t_{j_{\nu}-1}^{(n)}}{t_{j_{\nu}}^{(n)}-t_{j_{\nu}-1}^{(n)}} X_{n, j_{\nu}} \\
& -S_{n, i_{\nu}-1}-\frac{\nu / r-t_{i_{\nu}-1}^{(n)}}{t_{i_{\nu}}^{(n)}-t_{i_{\nu}-1}^{(n)}} X_{n, i_{\nu}}
\end{aligned}
$$

и, следовательно,

$$
\begin{aligned}
& \sqrt{2 s}\left|X_{n}(t)-X_{n}\left(\frac{\nu}{r}\right)\right| \leqslant\left|S_{n, j_{\nu}-1}-S_{n, i_{\nu}-1}\right|+\left|X_{n, i_{\nu}}\right|+\left|X_{n, j_{\nu}}\right| \\
& \quad=\left|S_{n, j_{\nu}-1}-S_{n, i_{\nu}-1}\right|+\left|X_{n, i_{\nu}}\right|+\left|S_{n, j_{\nu}}-S_{n, j_{\nu}-1}\right| \leqslant 4 \max _{i_{\nu} \leqslant k \leqslant j_{\nu}}\left|\sum_{i=i_{\nu}}^{k} X_{n, i}\right| .
\end{aligned}
$$

Такое же неравенство мы получим при других возможных расположениях чисел $\nu / r,(\nu+1) / r, t, t_{i_{\nu}}^{(n)}, t_{j_{\nu}}^{(n)}$. По неравенству (34)

$$
\mathbf{P}\left\{\max _{i_{\nu} \leqslant k \leqslant j_{\nu}}\left|\sum_{i=i_{\nu}}^{k} X_{n, i}\right| \geqslant \frac{\delta}{4} \sqrt{2 s}\right\} \leqslant \frac{256 C}{\delta^{4}}\left(\frac{j_{\nu}+1-i_{\nu}}{2 s}\right)^{2}
$$

и, в силу (38),

$$
\begin{aligned}
\limsup _{n \rightarrow \infty} \mathbf{P}_{n}\left(D_{\nu}^{r}\right) & \leqslant \limsup _{n \rightarrow \infty} \mathbf{P}\left\{\max _{i_{\nu} \leqslant k \leqslant j_{\nu}}\left|\sum_{i=i_{\nu}}^{k} X_{n, i}\right| \geqslant \frac{\delta}{4} \sqrt{2 s}\right\} \\
& \leqslant \frac{256 C}{\delta^{4}} \lim _{n \rightarrow \infty}\left(\frac{j_{\nu}+1-i_{\nu}}{2 s}\right)^{2}=\frac{64 C}{r^{2} \delta^{4}} .
\end{aligned}
$$

Ясно, что можно выбрать натуральное число $r=r(\varepsilon, \delta)$ настолько большим, что будет выполняться неравенство $\mathbf{P}_{n}\left(\cup_{\nu=0}^{r-1} D_{\nu}^{r}\right) \leqslant \sum_{\nu=0}^{r-1} \mathbf{P}_{n}\left(D_{\nu}^{r}\right) \leqslant$ $\varepsilon / 2$ для всех достаточно больших $n$. Для множества функций $A_{l}=$ $\{x:\|x\| \leqslant l\}$ из $C[0,1]$ по упомянутому неравенству (34) мы имеем $\mathbf{P}_{n}\left(A_{l}\right)=\mathbf{P}\left\{\max _{1 \leqslant k \leqslant s}\left|S_{n, k}\right| \leqslant l \sqrt{2 s}\right\} \geqslant 1-C /\left(4 l^{4}\right)$. Поэтому с помощью подходящего выбора числа $l$ можно добиться неравенства $\mathbf{P}_{n}(H) \geqslant 1-\varepsilon$ для всех достаточно больших $n$, где $H=A_{l} \cap \cap_{\nu=0}^{r-1} C_{\nu}^{r}$ и $C_{\nu}^{r}$ обозначает дополнение $D_{\nu}^{r}$. Разобьем прямоугольник $\{(t, x): 0 \leqslant t \leqslant 1$, $|x| \leqslant l\}$ прямыми, параллельными осям $t$ и $\boldsymbol{x}$ и проходящими через точки $(\nu / r, p l / q), \nu=0,1, \ldots, r, p=0, \pm 1, \ldots, \pm q$, где $q$ равно целой части числа $4 / \delta$. Ясно, что $H$ лежит в $\delta$-окрестности множества $X(\varepsilon, \delta)$ кусочно-линейных функций из $C[0,1]$ с вершинами, лежашими в узлах сетки $\{(\nu / r, p l / q): \nu=0,1, \ldots, r, p=0, \pm 1, \ldots, \pm q\}$. Последовательность $\left\{\mathbf{P}_{n}\right\}_{n \geqslant 1}$ слабо компактна, так как $\mathbf{P}_{n}(S(X(\varepsilon, \delta), \delta)) \geqslant \mathbf{P}_{n}(H) \geqslant 1-\varepsilon$ для всех достаточно больших $n$. 
Докажем теперь, что конечномерные распределения случайной ломаной слабо сходятся к соответствующим конечномерным распределениям винеровского процесса. Пусть дан набор чисел $t_{j}, j=1,2, \ldots, d$, удовлетворяющих условию $0=t_{0}<t_{1}<t_{2}<\cdots<t_{d} \leqslant 1$. Для каждого $n$ найдутся такие $k_{j}=k_{n, j}$, что $t_{k_{j}-1}^{(n)}<t_{j} \leqslant t_{k_{j}}^{(n)}, j=1,2, \ldots, d$. Ясно, что $k_{j} / s \rightarrow t_{j}$ при $n \rightarrow \infty$. По определению (37)

$$
X\left(t_{j}\right)=\frac{S_{n, k_{j}-1}}{\sqrt{2 s}}+\frac{t_{j}-t_{k_{j}-1}^{(n)}}{t_{k_{j}}^{(n)}-t_{k_{j}-1}^{(n)}} \frac{X_{n, k_{j}}}{\sqrt{2 s}}=\frac{S_{n, k_{j}}}{\sqrt{2 s}}+\frac{t_{j}-t_{k_{j}}^{(n)}}{t_{k_{j}}^{(n)}-t_{k_{j}-1}^{(n)}} \frac{X_{n, k_{j}}}{\sqrt{2 s}} .
$$

Из неравенств

$$
\begin{gathered}
\left|X_{n, k_{j}}\right| \leqslant \frac{n}{p_{k_{j}}}\left(\frac{\nu_{k_{j}}}{n}-p_{k_{j}}\right)^{2}+q_{k_{j}}, \\
\mathbf{P}\left\{\frac{n}{p_{k_{j}}}\left(\frac{\nu_{k_{j}}}{n}-p_{k_{j}}\right)^{2} \geqslant \lambda\right\} \leqslant \frac{q_{k_{j}}}{\lambda}, \quad \lambda>0,
\end{gathered}
$$

второе из которых выполняется по неравенству Маркова, следует, что $X_{n, k_{j}} / \sqrt{2 s}$ сходится по вероятности к нулю при $n \rightarrow \infty$, и, следовательно, разность $\left(X_{n}\left(t_{j}\right)-S_{n, k_{j}}\right) / \sqrt{2 s}$ также сходится по вероятности к нулю при $n \rightarrow \infty$. Поэтому

$$
\begin{aligned}
\lim _{n \rightarrow \infty}(\mathbf{P} & \left\{X_{n}\left(t_{j}\right)<x, j=1,2, \ldots, d\right\} \\
-\mathbf{P} & \left.\left\{\frac{S_{n, k_{j}}}{\sqrt{2 s}}<x, j=1,2, \ldots, d\right\}\right)=0 .
\end{aligned}
$$

Осталось заметить, что по теореме 3

$$
\begin{aligned}
& \lim _{n \rightarrow \infty} \mathbf{P}\left\{\frac{S_{n, k_{j}}}{\sqrt{\mathbf{D} \chi_{n}^{2}}} \sqrt{\frac{\mathbf{D} \chi_{n}^{2}}{2 s}}<x, j=1,2, \ldots, d\right\} \\
& =\mathbf{P}\left\{W\left(t_{j}\right)<x, j=1,2, \ldots, d\right\},
\end{aligned}
$$

так как $S_{n, k_{j}}=\chi_{n}^{2}\left(1, k_{j}\right)-\mathbf{E} \chi_{n}^{2}\left(1, k_{j}\right)$ и выполняется (15). Теорема доказана.

5. Слабая сходимость функционалов. Одним из следствий теоремы 4 является то, что для каждого почти непрерывного относительно меры Винера функционала $f(x), x \in C[0,1]$, последовательность случайных величин $\left\{f\left(X_{n}(t)\right)\right\}_{n \geqslant 1}$ слабо сходится $\mathrm{x}$ случайной величине $f(W(t))$. Здесь мы воспользуемся этим утверждением для доказательства слабой сходимости некоторых функционалов от частичных сумм 
суммы (1). Определим функции распределения $F, G$ и $G_{p}$, положив

$$
\begin{aligned}
F(x) & =\mathbf{P}\left\{\max _{0 \leqslant t \leqslant 1} W(t)<x\right\}=\left\{\begin{array}{cc}
\sqrt{\frac{2}{\pi}} \int_{0}^{x} \exp \left\{-\frac{u^{2}}{2}\right\} d u, & x>0, \\
0, & x \leqslant 0,
\end{array}\right. \\
G(x) & =\mathbf{P}\left\{\max _{0 \leqslant t \leqslant 1}|W(t)|<x\right\}=\frac{4}{\pi} \sum_{m=0}^{\infty} \frac{(-1)^{m}}{2 m+1} \exp \left\{-\frac{(2 m+1)^{2} \pi^{2}}{8 x^{2}}\right\}, \\
G_{p}(x) & =\mathbf{P}\left\{\int_{0}^{1}|W(t)|^{p} d t<x\right\}, \quad p>0, x \in \mathbf{R}^{1} .
\end{aligned}
$$

По поводу вычислений первых двух из этих функций распределения см. [12] и [13]. В [12] также приведены явные выражения функции $G_{p}(x)$ при $p=1$ и $p=2$. Нетрудно убедиться, что приведенные выше функции распределения непрерывны.

Далее окажется полезным одно элементарное неравенство. Пусть дано положительное число $p$. Запишем его в виде суммы $p=q+h$ его целой части $q$ и его дробной части $h$. Для любых положительных чисел $a$ и $b$ справедливо неравенство

$$
\left|a^{p}-b^{p}\right| \leqslant \begin{cases}p|a-b| \max _{0 \leqslant l<q}\left\{a^{l+h} b^{q-l-1}\right\}+b^{q}|a-b|^{h}, & h>0, \\ p|a-b| \max _{0 \leqslant l<p}\left\{a^{l} b^{p-l-1}\right\}, & h=0,\end{cases}
$$

где максимум величин полагается равным нулю при $q=0$ и $l$ принимает целые значения.

Докажем это неравенство. Если $0<p<1$, то $a^{p}-b^{p}=|a-b+b|^{p}-$ $b^{p} \leqslant|a-b|^{p}+b^{p}-b^{p}=|a-b|^{p}$. В этих выражениях числа $a$ и $b$ можно поменять ролями. Из этих соотношений следует объявленное неравенство при $0<p<1$. Если $p \geqslant 1$, то $a^{p}-b^{p}=\left(a^{q}-b^{q}\right) a^{h}+b^{q}\left(a^{h}-b^{h}\right)$ и $\left|b^{a}-b^{q}\right|=$ $|a-b|\left(a^{q-1}+a^{q-2} b+\cdots+a b^{q-2}+b^{q-1}\right) \leqslant q|a-b| \max _{0 \leqslant l<q}\left\{a^{l} b^{q-l-1}\right\}$. И3 этих соотношений следует объявленное неравенство.

Tеорема 5. Предположим, что величины $s u p_{k}, k=1,2, \ldots, s, u$ множество $W \subset\{1,2, \ldots, s\}$ зависят от $n$ тахим образом, ито $s \rightarrow \infty$ при $n \rightarrow \infty$ и выполнены условия (6)-(8). Тогда для любого $x \in \mathbf{R}^{1}$

$$
\begin{array}{r}
\lim _{n \rightarrow \infty} \mathbf{P}\left\{\max _{1 \leqslant k \leqslant s} \sum_{i=1}^{k}\left(\frac{n}{p_{i}}\left(\frac{\nu_{i}}{n}-p_{i}\right)^{2}-q_{i}\right) \frac{1}{\sqrt{\mathbf{D} \chi_{n}^{2}}}<x\right\}=F(x), \\
\lim _{n \rightarrow \infty} \mathbf{P}^{\prime}\left\{\max _{1 \leqslant k \leqslant s}\left|\sum_{i=1}^{k}\left(\frac{n}{p_{i}}\left(\frac{\nu_{i}}{n}-p_{i}\right)^{2}-q_{i}\right)\right| \frac{1}{\sqrt{\mathbf{D} \chi_{n}^{2}}}<x\right\}=G(x), \\
\lim _{n \rightarrow \infty} \mathbf{P}\left\{\frac{2}{\left(\mathbf{D} \chi_{n}^{2}\right)^{(p+2) / 2}} \sum_{k=1}^{s}\left|\sum_{i=1}^{k}\left(\frac{n}{p_{i}}\left(\frac{\nu_{i}}{n}-p_{i}\right)^{2}-q_{i}\right)\right|^{p}<x\right\}=G_{p}(x),
\end{array}
$$

где $p$ - мюбое положительное число. 
Д ок а з а т е л ь с т в о. Утверждение (40) является следствием теоремы 4 и соотношения (15), так как

$$
\max _{1 \leqslant k \leqslant s} \sum_{i=1}^{k}\left(\frac{n}{p_{i}}\left(\frac{\nu_{i}}{n}-p_{i}\right)^{2}-q_{i}\right) \frac{1}{\sqrt{\mathbf{D} \chi_{n}^{2}}}=\max _{0 \leqslant t \leqslant 1} X_{n}(t) \sqrt{\frac{2 s}{\mathbf{D} \chi_{n}^{2}}}
$$

и последовательность случайных величин $\left\{\max _{0 \leqslant t \leqslant 1} X_{n}(t)\right\}_{n \geqslant 1}$ слабо сходится к случайной величине $\max _{0 \leqslant t \leqslant 1} W(t)$. Аналогично можно доказать утверждение (41).

Докажем (42). По теореме $4 \lim _{n \rightarrow \infty} \mathbf{P}\left\{\int_{0}^{1}\left|X_{n}(t)\right|^{p} d t<x\right\}=$ $\mathbf{P}\left\{\int_{0}^{1}|W(t)|^{p} d t<x\right\}$. В силу (37)

$$
\begin{aligned}
& \int_{0}^{1}\left|X_{n}(t)\right|^{p} d t=\frac{1}{(2 s)^{p / 2}} \sum_{k=1}^{s} \int_{t_{k-1}^{(n)}}^{t_{k}^{(n)}}\left|S_{n, k-1}+\frac{t-t_{k-1}^{(n)}}{t_{k}^{(n)}-t_{k-1}^{(n)}} X_{n, k}\right|^{p} d t \\
& =\frac{1}{(2 s)^{p / 2}} \sum_{k=1}^{s} \int_{t_{k-1}^{(n)}}^{t_{k}^{(n)}}\left|S_{n, k}+\frac{t-t_{k}^{(n)}}{t_{k}^{(n)}-t_{k-1}^{(n)}} X_{n, k}\right|^{p} d t \\
& =\frac{2}{(2 s)^{(p+2) / 2}} \sum_{k=1}^{s}\left|S_{n, k}\right|^{p} \\
& \quad+\frac{1}{(2 s)^{p / 2}} \sum_{k=1}^{s} \int_{t_{k-1}^{(n)}}^{t_{k}^{(n)}}\left(\left|S_{n, k}+\frac{t-t_{k}^{(n)}}{t_{k}^{(n)}-t_{k-1}^{(n)}} X_{n, k}\right|^{p}-\left|S_{n, k}\right|^{p}\right) d t .
\end{aligned}
$$

Из этих соотношений будет следовать

$$
\lim _{n \rightarrow \infty} \mathbf{P}\left\{\frac{2}{(2 s)^{(p+2) / 2}} \sum_{k=1}^{s}\left|S_{n, k}\right|^{p}<x\right\}=\mathbf{P}\left\{\int_{0}^{1}|W(t)|^{p} d t<x\right\},
$$

если $J_{n}$ сходится по вероятности к нулю при $n \rightarrow \infty$, где

$$
J_{n}=\frac{1}{(2 s)^{p / 2}} \sum_{k=1}^{s} \int_{t_{k-1}^{(n)}}^{t_{k}^{(n)}}\left(\left|S_{n, k}+\frac{t-t_{k}^{(n)}}{t_{k}^{(n)}-t_{k-1}^{(n)}} X_{n, k}\right|^{p}-\left|S_{n, k}\right|^{p}\right) d t .
$$

Утверждения (42) и (43) эквивалентны в силу соотношения (15). Оценим величину $J_{n}$. Справедливо следующее неравенство:

$$
\left|J_{n}\right| \leqslant \frac{1}{(2 s)^{p / 2}} \sum_{k=1}^{s} \int_{t_{k-1}^{(n)}}^{t_{k}^{(n)}} I_{n} d t
$$

где

$$
I_{n}=|| S_{n, k}+\left.\frac{t-t_{k}^{(n)}}{t_{k}^{(n)}-t_{k-1}^{(n)}} X_{n, k}\right|^{p}-\left|S_{n, k}\right|^{p} \mid
$$


Далее рассуждения будут зависеть от величины $p$. Рассмотрим сначала случай, когда $p>1$ и $h>0$. По неравенству (39)

$$
\begin{aligned}
I_{n} & \leqslant p\left|X_{n, k}\right| \max _{0 \leqslant l<q}\left\{\left|S_{n, k}+\frac{t-t_{k}^{(n)}}{t_{k}^{(n)}-t_{k-1}^{(n)}} X_{n, k}\right|^{l+h}\left|S_{n, k}\right|^{q-l-1}\right\}+\left|S_{n, k}\right|^{q}\left|X_{n, k}\right|^{h} \\
& \leqslant p\left|X_{n, k}\right| \max _{0 \leqslant l<q}\left\{2^{l+h}\left(\left|S_{n, k}\right|^{l+h}+\left|X_{n, k}\right|^{l+h}\right)\left|S_{n, k}\right|^{q-l-1}\right\}+\left|S_{n, k}\right|^{q}\left|X_{n, k}\right|^{h}
\end{aligned}
$$

Здесь мы воспользовались элементарным неравенством $(a+b)^{\alpha} \leqslant 2^{\alpha}\left(a^{\alpha}+\right.$ $\left.b^{\alpha}\right)$, справедливым для любых положительных чисел $a, b$ и $\alpha$. Если в предыдущем неравенстве заменить сумму $S_{n, k}$ максимальной суммой и принять во внимание, что $X_{n, k}=S_{n, k}-S_{n, k-1}$ и $l+h \leqslant p$, то оденка для $I_{n}$ будет иметь вид

$$
I_{n} \leqslant 6^{p} p\left|X_{n, k}\right|\left(\max _{1 \leqslant k \leqslant s}\left|S_{n, k}\right|\right)^{p-1}+\left(\max _{1 \leqslant k \leqslant s}\left|S_{n, k}\right|\right)^{q}\left|X_{n, k}\right|^{h},
$$

и, в силу (44),

$$
\begin{aligned}
\left|J_{n}\right| \leqslant & \frac{1}{(2 s)^{p / 2}} \sum_{k=1}^{s} \int_{t_{k-1}^{(n)}}^{t_{k}^{(n)}}\left(6^{p} p\left(\max _{1 \leqslant k \leqslant s}\left|S_{n, k}\right|\right)^{p-1}\left|X_{n, k}\right|\right. \\
& \left.+\left(\max _{1 \leqslant k \leqslant s}\left|S_{n, k}\right|\right)^{q}\left|X_{n, k}\right|^{h}\right) d t \\
= & 6^{p} p\left(\frac{\max _{1 \leqslant k \leqslant s}\left|S_{n, k}\right|}{\sqrt{2 s}}\right)^{p-1} \frac{2}{(2 s)^{3 / 2}} \sum_{k=1}^{s}\left|X_{n, k}\right| \\
& +\left(\frac{\max _{1 \leqslant k \leqslant s}\left|S_{n, k}\right|}{\sqrt{2 s}}\right)^{q} \frac{2}{(2 s)^{(2+h) / 2}} \sum_{k=1}^{s}\left|X_{n, k}\right|^{h} .
\end{aligned}
$$

С помощью неравенства (34) легко убедиться, что последовательность случайных величин $\left\{\max _{1 \leqslant k \leqslant s}\left|S_{n, k}\right| / \sqrt{2 s}\right\}_{n \geqslant 1}$ слабо компактна. Из предыдущего неравенства следует, что $J_{n}$ сходится по вероятности к нулю при $n \rightarrow \infty$, если

$$
\begin{aligned}
\lim _{n \rightarrow \infty} \mathbf{P}\left\{\frac{1}{(2 s)^{3 / 2}} \sum_{k=1}^{s}\left|X_{n, k}\right| \geqslant \varepsilon\right\} & =0, \\
\lim _{n \rightarrow \infty} \mathbf{P}\left\{\frac{1}{(2 s)^{(2+h) / 2}} \sum_{k=1}^{s}\left|X_{n, k}\right|^{h} \geqslant \varepsilon\right\} & =0, \quad \varepsilon>0 .
\end{aligned}
$$

Докажем, что оба соотношения выполняются. Рассмотрим, например, второе из них. По неравенству Маркова

$$
\begin{aligned}
\mathbf{P}\left\{\frac{1}{(2 s)^{(2+h) / 2}} \sum_{k=1}^{s}\left|X_{n, k}\right|^{h} \geqslant \varepsilon\right\} & \leqslant \frac{1}{\varepsilon(2 s)^{(2+h) / 2}} \sum_{k=1}^{s} \mathbf{E}\left|X_{n, k}\right|^{h} \\
& \leqslant \frac{1}{\varepsilon(2 s)^{h / 2}} \max _{1 \leqslant k \leqslant s} \mathbf{E}\left|X_{n, k}\right|^{h} .
\end{aligned}
$$


Из соотношений

$$
\begin{aligned}
\mathbf{E}\left|X_{n, k}\right|^{h} & \leqslant \mathbf{E}\left(\frac{n}{p_{k}}\left(\frac{\nu_{k}}{n}-p_{k}\right)^{2}\right)^{h}+q_{k}^{h} \\
& \leqslant\left(\mathbf{E}\left(\frac{n}{p_{k}}\left(\frac{\nu_{k}}{n}-p_{k}\right)^{2}\right)\right)^{h}+q_{k}^{h}=2 q_{k}^{h} \leqslant 2
\end{aligned}
$$

где второе неравенство выполняется по неравенству Маркова для абсолютных моментов, следует, что

$$
\lim _{n \rightarrow \infty} \mathbf{P}\left\{\frac{1}{(2 s)^{(2+h) / 2}} \sum_{k=1}^{s}\left|X_{n, k}\right|^{h} \geqslant \varepsilon\right\} \leqslant \lim _{n \rightarrow \infty} \frac{2}{(2 s)^{h / 2}}=0 .
$$

Первое соотношение также выполняется. Достаточно в разобранном случае положить $h=1$. Теорема доказана в случае, если $p>1$ и $h>0$. Проведенные рассуждения фактически содержат доказательство теоремы в случае $p>1$ и $h=0$. В случае $p \leqslant 1$ справедливы оценки $\left|I_{n}\right| \leqslant\left|X_{n, k}\right|^{p}$ и $\left|J_{n}\right| \leqslant 2(2 s)^{-(p+2) / 2} \sum_{k=1}^{s}\left|X_{n, k}\right|^{p}$. Выше мы видели, что правая часть этого неравенства сходится по вероятности к нулю при $n \rightarrow \infty$. Теорема доказана.

Прием, с помощью которого теорема 2 была выведена из теоремы 1 , позволяет вывести следующее утверждение из только что доказанной теоремы.

Теорема 6. Предположим, что множество $W=\{r, r+1, \ldots, l\} \subset$ $\{1,2, \ldots, s\}$, числа $r, l, 1 \leqslant r<l \leqslant s$, и величины $p_{k}, k \in W$, зависят от $n$ таким образом, что $(l-r) \rightarrow \infty$ при $n \rightarrow \infty$ и выполнено условие (7). Тогда

$$
\begin{array}{r}
\lim _{n \rightarrow \infty} \mathbf{P}\left\{\max _{r \leqslant k \leqslant l} \sum_{i=r}^{k}\left(\frac{n}{p_{i}}\left(\frac{\nu_{i}}{n}-p_{i}\right)^{2}-q_{i}\right) \frac{1}{\left.\sqrt{\mathbf{D} \chi_{n}^{2}(W)}<x\right\}}=F(x),\right. \\
\lim _{n \rightarrow \infty} \mathbf{P}\left\{\max _{r \leqslant k \leqslant l}\left|\sum_{i=r}^{k}\left(\frac{n}{p_{i}}\left(\frac{\nu_{i}}{n}-p_{i}\right)^{2}-q_{i}\right)\right| \frac{1}{\left.\sqrt{\mathbf{D} \chi_{n}^{2}(W)}<x\right\}=G(x),}\right. \\
\lim _{n \rightarrow \infty} \mathbf{P}\left\{\frac{2}{\left(\mathbf{D} \chi_{n}^{2}(W)\right)^{(p+2) / 2}} \sum_{k=r}^{l}\left|\sum_{i=r}^{k}\left(\frac{n}{p_{i}}\left(\frac{\nu_{i}}{n}-p_{i}\right)^{2}-q_{i}\right)\right|^{p}<x\right\}=G_{p}(x),
\end{array}
$$

где р - любое положительное число.

В заключение автор выражает глубокую благодарность В. П. Чистякову и рецензентам, чьи советы способствовали улучпению первоначального текста. В частности, они любезно указали на статьи [14]-[17], имеющие близкое отношение к изложенному. 


\section{СПИСОК ЛИТЕРАТУРЫ}

1. Крамер Г. Математические методы статистики. М.: Мир, 1975, 648 с.

2. Тумандя C.X. Асимптотическое распределение критерия $\chi^{2}$ при одновремевном возрастании объема наблюдений и числа групп. - Теория вероятн. и ее примен., 1956 , т. I, в. 1 , с. $131-145$.

3. Гихмая И.И. Об асимптотических свойствах некоторых статистик, аналогичных величине $\chi^{2}$. - Теория вероятн. и ее примен., 1956, т. I, в. 3, с. 344-348.

4. Ивъенжо Г.И., Левия В.В. Разделимые статистики и критерии однородности для полиномиальных выборок. - Обозрение прикл. промышл. матем., сер. дискретн. матем., 1995, т. 2, в. 6, с. 871-887.

5. Ермаков $M$. . А. Асимптотическая минимаксность критериев хи-квадрат. - Теория вероятн. и ее примен., 1997, т. 42, в. 4; с. 668-695.

6. Morris C. Central limit theorems for multinomial sums. - Ann. Statist., 1975, v. 3, № 1, p. 165-188.

7. Круглов В.М. К приншипу ипвариантности. - Теория вероятн. и ее примен., 1997 , т. 42 , в. 2 , с. $239-261$.

8. Курош А. Г. Курс высшей алгебры. М.: Наука, 1968, 431 с.

9. Биллиягсли П. Сходимость вероятностных мер. М.: Наука, 1977, 351 с.

10. Haldan $J$. The exact value of the moments of the distribution of $\chi^{2}$ used as a test of goodness of fit, when expectations are small. - Biometrics, 1937, v. 29, p. 133-143.

11. Прохоров Ю. В. Сходимость случайных процессов и предельные теоремқ теории вероятностей. - Теория вероятн. и ее примен., 1956, т. I, в. 2, с. 176-238.

12. Erdos $P$., Kac $M$. On certain limit theorems in the theory of probability. - Bull. Amer. Math. Soc., 1946, v. 52, p. 292-302.

13. Donsker M.D. An invariance principle for certain probability limit theorems. Mem. Amer. Math. Soc., 1951, № 6, p. 1-12.

14. Медведев Ю. И. Некоторые теоремы об асимптотическом распределении статистики $\chi^{2}$. - Докл. АН СССР, 1970, т. 192, № 5, с. 987-989.

15. Медведев Ю.И. Разделимые статистики в полиномиальной схеме, I. - Теория вероятн. и ее примен., 1977 , т. XXII, в. 1, с. 3-17.

16. Медведев Ю.И. Разделимые статистики в полшномиальной схеме, II. - Теория вероятн. и ее примен., 1977 , т. XXII, в. 3, с. 623-631.

17. Захаров В.K., Сарманов О. В., Севастьянов Б. А. Последовательный критерий $\chi^{2}$. Матем. с6., 1969 , т. 79 , № 3, с. 444-460. 